Supporting Information for

\title{
Hydrodeoxygenative Cyclotetramerization of Carbon Monoxide by a Trinuclear Titanium Polyhydride Complex
}

\author{
Shaowei $\mathrm{Hu}^{\dagger \S}$, Takanori Shima ${ }^{\dagger *}$, and Zhaomin $\mathrm{Hou}^{* \dagger}$ \\ †Organometallic Chemistry Laboratory, RIKEN Cluster for Pioneering Research, 2-1 Hirosawa, \\ Wako, Saitama 351-0198, Japan \\ ¥Advanced Catalysis Research Group, RIKEN Center for Sustainable Resource Science, 2-1 \\ Hirosawa, Wako, Saitama 351-0198, Japan \\ *houz@riken.jp \\ §Present Address: College of Chemistry, Beijing Normal University, No. 19, Xinwai street, Beijing \\ 100875, People's Republic of China.
}

\section{Contents}

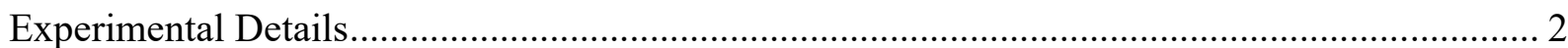

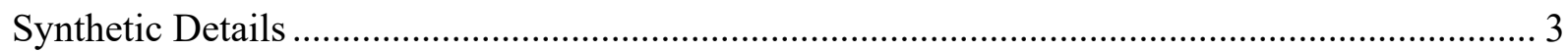

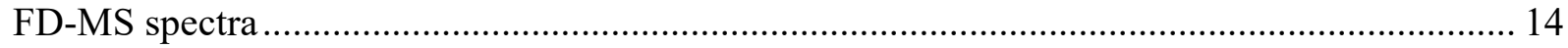

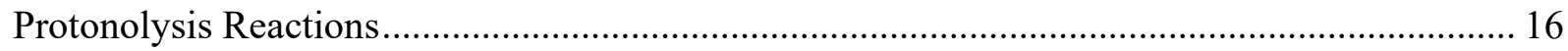

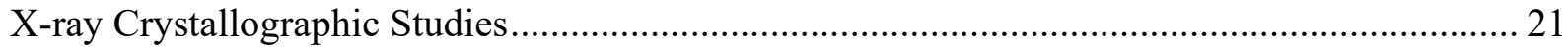

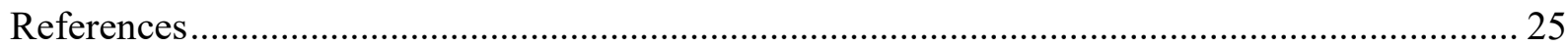




\section{Experimental Details}

\section{General Considerations}

All reactions were carried out under a dry and oxygen-free argon atmosphere by using Schlenk techniques or under an argon atmosphere in an Mbraun glovebox. The argon was purified by being passed through a Drycolumn DC-A4 (Nikka Seiko Co.) and a Gascolumn GC-RX (Nikka Seiko Co.). The argon in the glovebox was constantly circulated through a copper catalyst/activated charcoal unit. The oxygen and moisture concentrations in the glovebox atmosphere were monitored by an $\mathrm{O}_{2} / \mathrm{H}_{2} \mathrm{O}$ Combi-Analyzer (Mbraun) to ensure both were always below $1 \mathrm{ppm}$. Samples for NMR spectroscopic measurements were prepared in the glovebox by use of J. Young valve NMR tubes. ${ }^{1} \mathrm{H}$ and ${ }^{13} \mathrm{C}$ NMR spectrum were recorded on a JEOL JNM-ECS400, or a Bruker AVANCE III HD 500 spectrometer. Elemental analyses were performed by a MICRO CORDER JM10. Field desorption (FD) mass spectra were acquired on a JEOL JMS-T100GCV time of flight mass spectrometer equipped with an EI/FI(FD) combination ion source. Anhydrous THF, hexane, benzene, and toluene were purified by use of a SPS- 800 solvent purification system (Mbraun), and dried over fresh Na chips in the glovebox. NMR solvents were dried with activated $3 \AA$ molecular sieves. $\left[\left(\mathrm{C}_{5} \mathrm{Me}_{4} \mathrm{SiMe}_{3}\right)_{3} \mathrm{Ti}_{3}\left(\mu_{3}-\mathrm{H}\right)\left(\mu_{2}-\mathrm{H}\right)_{6}\right](\mathbf{1})$ was prepared according to literature procedures ${ }^{1}$ and was stored in the glovebox. Hydrogen $(>99.99999 \%)$, CO $(>99.95 \%)$ were purchased from TAIYO NIPPON SANSO and used as received. $\mathrm{D}_{2}(99.96 \mathrm{~atm} \%),{ }^{13} \mathrm{CO}(99 \mathrm{~atm} \%)$ were purchased from SHOKO science. Ultrapure water was purchased from Wako and was degassed and stored under Ar atmosphere. Other chemicals were purchased from common commercial sources and used as received. 


\section{Synthetic Details}

$\left[\left(\mathrm{C}_{5} \mathrm{Me}_{4} \mathrm{SiMe}_{3}\right)_{3} \mathrm{Ti}_{3}\left(\mu-\eta^{1}: \eta^{1}: \eta^{2}-\mathbf{C H C H}\right)\left(\mu_{2}-\mathbf{O C H}_{3}\right)\left(\mu_{2}-\mathrm{O}\right)_{2}\right]$ (2). A THF solution (3.0 mL) of $\mathbf{1}$ (100 mg, $0.137 \mathrm{mmol})$ in $20 \mathrm{~mL}$ Schlenk tube equipped with a J. Young valve was frozen in liquid nitrogen, pumped, and backfilled with $\mathrm{CO}$. The solution was stirred at $-78{ }^{\circ} \mathrm{C}$ for $10 \mathrm{~min}$. The solution turned from dark green to dark red-brown. After removal of the solvent under vacuum, the resulting solid was dissolved in hexane, concentrated and crystallized at $-33^{\circ} \mathrm{C}$ to give a dark brown crystals $(87 \mathrm{mg})$ which contain $2(0.082 \mathrm{mmol}, 60 \%)$ and minor species $(0.027 \mathrm{mmol}, 20 \%)$. Attempts to separate 2 from the minor species by repeated recrystallization in various solvents did not give a pure product. Single crystals of 2 suitable for X-ray diffraction studies were obtained by recrystallization from a hexane solution at $-33{ }^{\circ} \mathrm{C}$. The preparation of the ${ }^{13} \mathrm{C}$-enriched compound $\left[\left(\mathrm{C}_{5} \mathrm{Me}_{4} \mathrm{SiMe}_{3}\right)_{3} \mathrm{Ti}_{3}\left(\mu-\eta^{1}: \eta^{1}: \eta^{2}{ }^{13} \mathrm{CH}^{13} \mathrm{CH}\right)\left(\mu_{2}-\mathrm{O}^{13} \mathrm{CH}_{3}\right)\left(\mu_{2}-\mathrm{O}\right)_{2}\right]\left(2-{ }^{13} \mathrm{C}_{3}\right)$ was carried out in the same manner by using ${ }^{13} \mathrm{CO}$.

2: ${ }^{1} \mathrm{H}$ NMR (400 MHz, THF-d8, rt): 7.88 (s, $\left.1 \mathrm{H}, \mathrm{C}_{2} \mathrm{H}_{2}\right), 7.53\left(\mathrm{~s}, 1 \mathrm{H}, \mathrm{C}_{2} \mathrm{H}_{2}\right), 4.07$ (s, 3H, OCH3), 2.01-1.56 (s, 6H×6, $\left.\mathrm{C}_{5} \mathrm{Me}_{4} \mathrm{SiMe}_{3}\right), 0.31$ (s, 18H, $\left.\mathrm{C}_{5} \mathrm{Me}_{4} \mathrm{SiMe}_{3}\right), 0.24$ (s, 9H, $\left.\mathrm{C}_{5} \mathrm{Me}_{4} \mathrm{SiMe}_{3}\right) .{ }^{1} \mathrm{H}$ NMR (400 MHz, THF- $\left.d 8,-40{ }^{\circ} \mathrm{C}\right): 7.89\left(\mathrm{~d}, J_{\mathrm{H}, \mathrm{H}}=12.0 \mathrm{~Hz}, 1 \mathrm{H}, \mathrm{C}_{2} H_{2}\right), 7.54\left(\mathrm{~d}, J_{\mathrm{H}, \mathrm{H}}=12.0 \mathrm{~Hz}\right.$, $\left.1 \mathrm{H}, \mathrm{C}_{2} \mathrm{H}_{2}\right), 4.08\left(\mathrm{~s}, 3 \mathrm{H}, \mathrm{OCH}_{3}\right), 2.02-1.55\left(\mathrm{~s}, 6 \mathrm{H} \times 6, \mathrm{C}_{5} \mathrm{Me}_{4} \mathrm{SiMe}_{3}\right), 0.31$ (s, 18H, $\left.\mathrm{C}_{5} \mathrm{Me}_{4} \mathrm{SiMe}_{3}\right)$, 0.24 (s, 9H, $\left.\mathrm{C}_{5} \mathrm{Me}_{4} \mathrm{SiMe} 3\right) .{ }^{13} \mathrm{C}$ NMR (100 MHz, THF-d8, rt): $222.4\left(\mathrm{~s}, \mathrm{C}_{2} \mathrm{H}_{2}\right), 210.2\left(\mathrm{~s}, \mathrm{C}_{2} \mathrm{H}_{2}\right)$, 128.8-123.9 (s, $C_{5} \mathrm{Me}_{4} \mathrm{SiMe}_{3}$ ), 119.8-115.5 (s, ipso- $C_{5} \mathrm{Me}_{4} \mathrm{SiMe}_{3}$ ), 65.6 (s, OCH3), 15.4-11.7 (s, $\mathrm{C}_{5} \mathrm{Me}_{4} \mathrm{SiMe}_{3}$ ), 2.8-2.1 (s, $\mathrm{C}_{5} \mathrm{Me}_{4} \mathrm{Si}_{2} e_{3}$ ). Anal. Calcd for $\mathrm{C}_{39} \mathrm{H}_{68} \mathrm{O}_{3} \mathrm{Si}_{3} \mathrm{Ti}_{3}$ : C, 57.63; H, 8.43. Found: C, 57.98; H, 8.43.

2- $^{13} \mathrm{C}_{3}:{ }^{1} \mathrm{H}$ NMR $\left(400 \mathrm{MHz}, \mathrm{THF}-d_{8}, \mathrm{rt}\right): 7.70\left(\mathrm{t}, J=143.7 \mathrm{~Hz}, 2 \mathrm{H},{ }^{13} \mathrm{C}_{2} \mathrm{H}_{2}\right), 4.07(\mathrm{~d}, J=141.4 \mathrm{~Hz}$, $\left.\mathrm{O}^{13} \mathrm{CH}_{3}\right), \quad 2.01-1.58 \quad\left(\mathrm{~s}, 6 \mathrm{H} \times 6, \mathrm{C}_{5} \mathrm{Me}_{4} \mathrm{SiMe}_{3}\right), 0.31 \quad\left(\mathrm{~s}, \quad 18 \mathrm{H}, \mathrm{C}_{5} \mathrm{Me}_{4} \mathrm{SiMe}_{3}\right), 0.24 \quad(\mathrm{~s}, 9 \mathrm{H}$, 
$\left.\mathrm{C}_{5} \mathrm{Me}_{4} \mathrm{Si}_{2} e_{3}\right) .{ }^{13} \mathrm{C}$ NMR (100 MHz, THF-d8, rt) (partially characterized): 222.4 (br.s, ${ }^{13} \mathrm{C}_{2} \mathrm{H}_{2}$ ), 210.2 (br.s, $\left.{ }^{13} \mathrm{C}_{2} \mathrm{H}_{2}\right), 65.6\left(\mathrm{~s}, \mathrm{O}^{13} \mathrm{CH}_{3}\right)$.

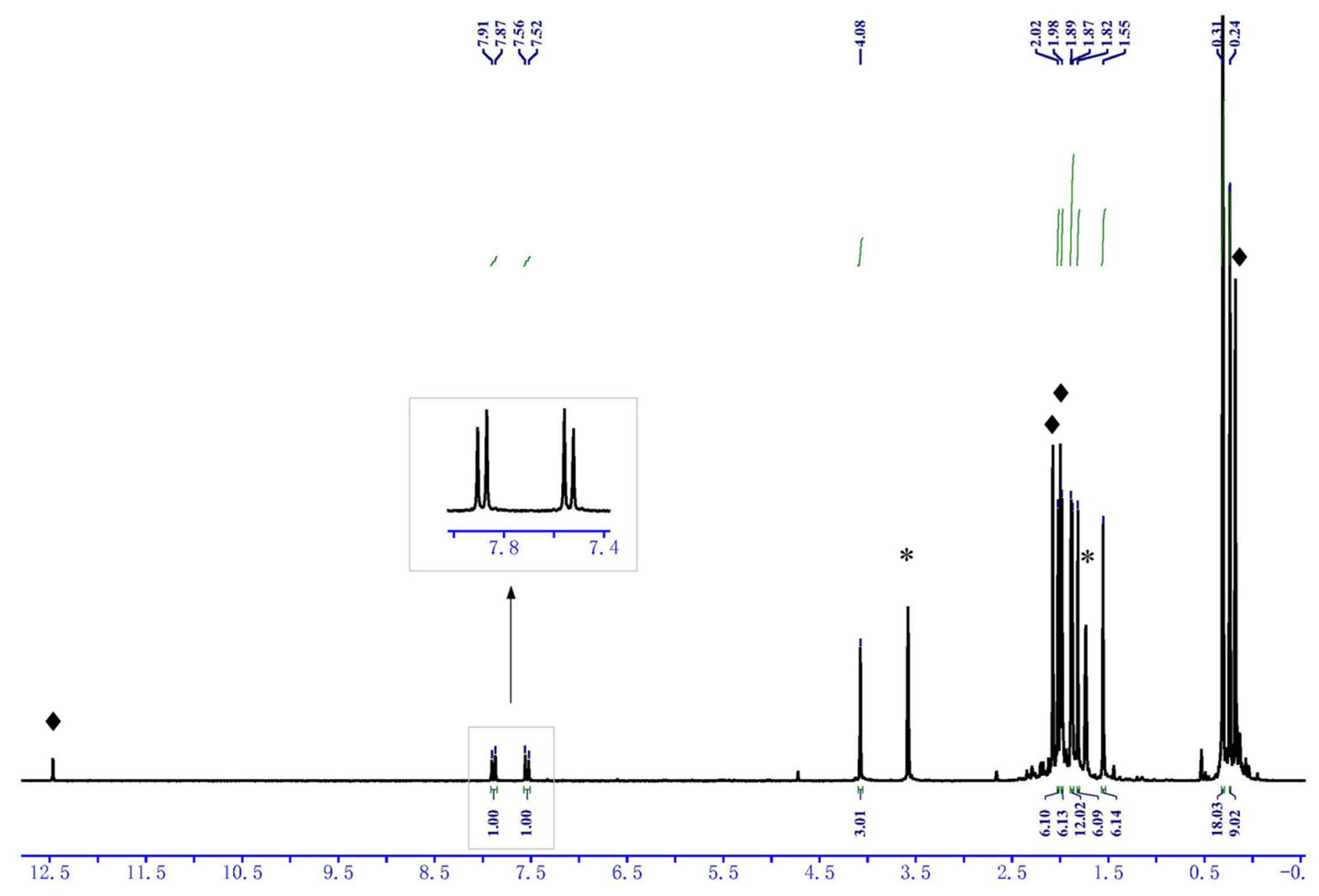

Figure S1. ${ }^{1} \mathrm{H}$ NMR spectrum of $2\left(\mathrm{THF}-d_{8},-40{ }^{\circ} \mathrm{C}, * \cdots\right.$ residual internal $\mathrm{C}_{4} \mathrm{D}_{7} \mathrm{HO}, \cdots$ minor species) 


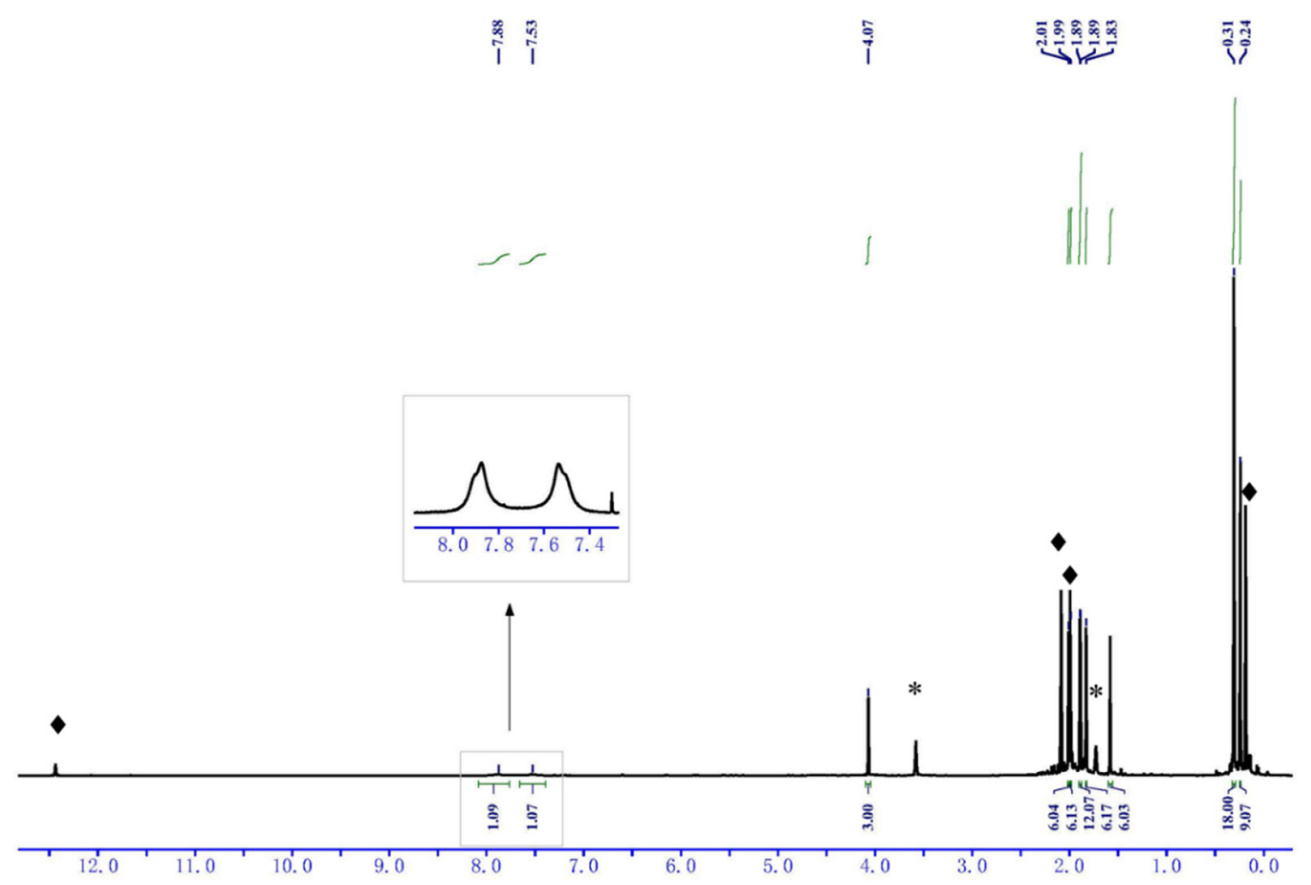

Figure S2. ${ }^{1} \mathrm{H}$ NMR spectrum of $2\left(\mathrm{THF}-d_{8}, \mathrm{rt}, * \cdots\right.$ residual internal $\mathrm{C}_{4} \mathrm{D}_{7} \mathrm{HO}, \cdots$ minor species)

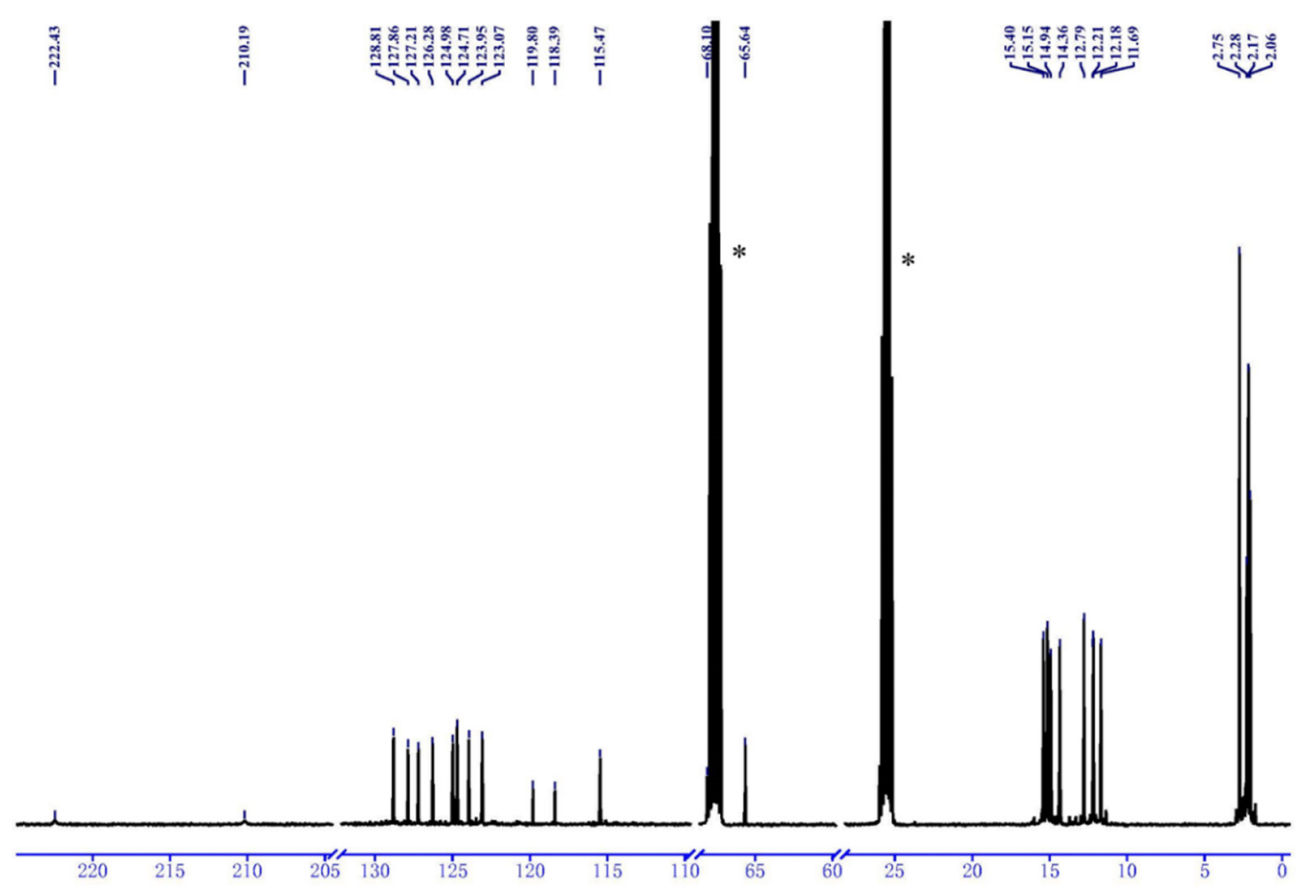

Figure S3. ${ }^{13} \mathrm{C}$ NMR spectrum of 2 (THF- $\left.d_{8}, \mathrm{rt}, * \cdots \mathrm{THF}-d_{8}\right)$ 


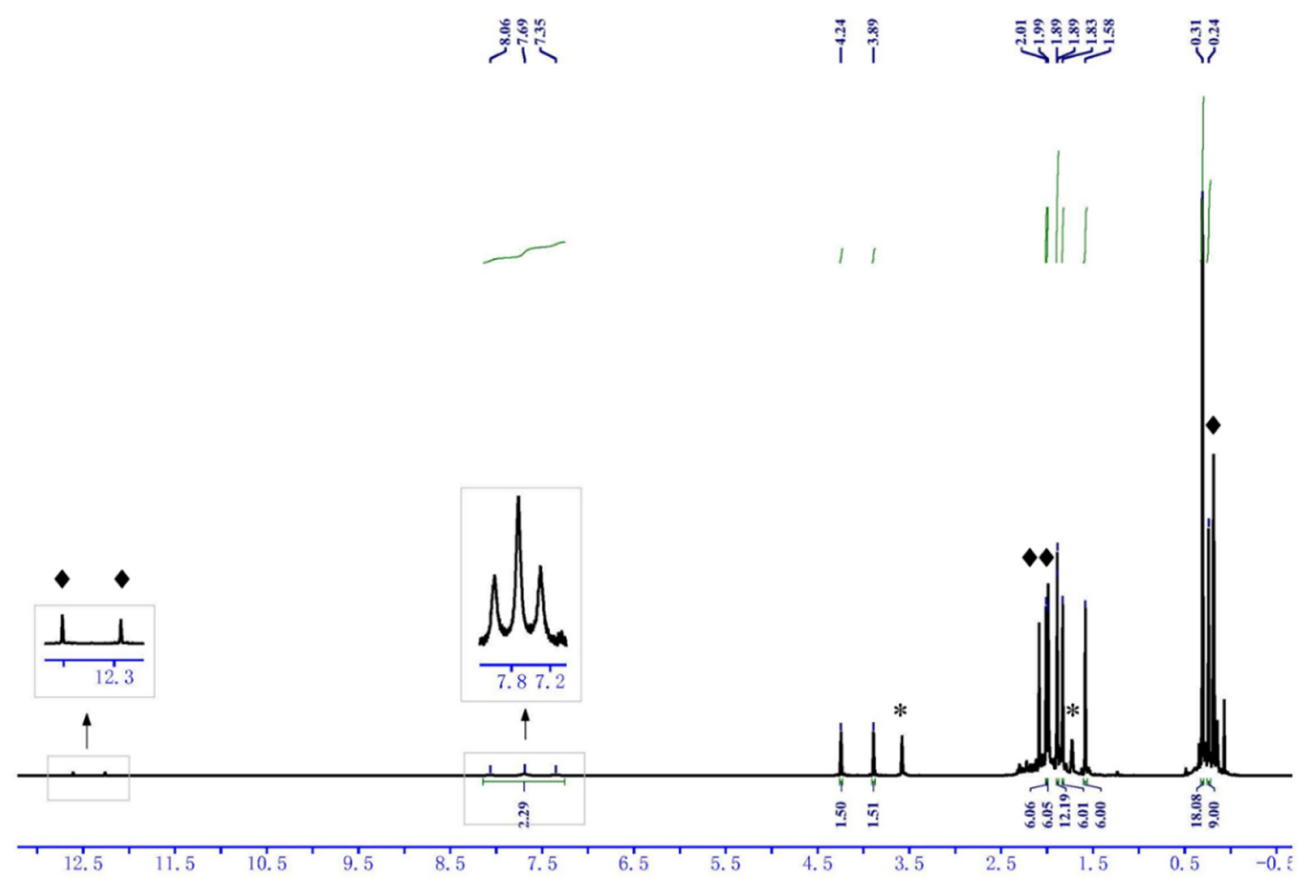

Figure S4. ${ }^{1} \mathrm{H}$ NMR spectrum of $\mathbf{2}^{-13} \mathbf{C}_{3}\left(\mathrm{THF}-d 8\right.$, rt, ${ }^{* \cdots}$ residual internal $\mathrm{C}_{4} \mathrm{D} 7 \mathrm{HO}, \cdots$ minor species)
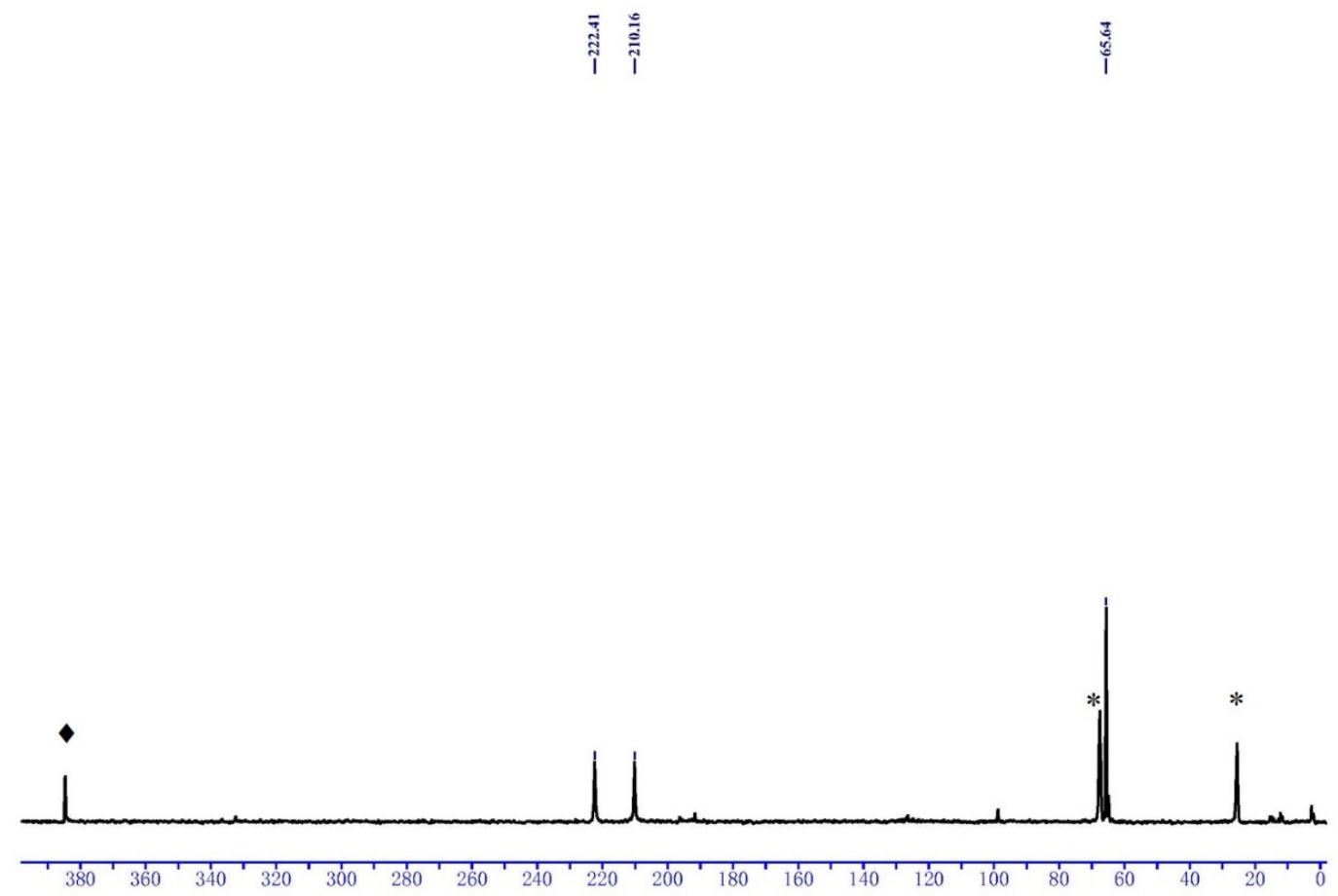

Figure S5. ${ }^{13} \mathrm{C}$ NMR spectrum of $2-{ }^{13} \mathbf{C}_{3}$ (THF- $d 8$, rt, $* \cdots$ THF- $d 8, \cdots$ minor species) 
$\left[\left(\mathrm{C}_{5} \mathrm{Me}_{4} \mathrm{SiMe}_{3}\right)_{3} \mathrm{Ti}_{3}\left(\mu-\eta^{1}: \eta^{1}: \eta^{2}-\mathbf{C}_{4} \mathrm{H}_{2} \mathrm{O}_{2}\right)\left(\mu_{2}-\mathbf{O C H}_{3}\right)\left(\mu_{2}-\mathbf{O}\right)_{2}\right]$ (3). A THF solution (3.0 mL) of 2 (100 mg, $0.123 \mathrm{mmol})$ in $20 \mathrm{~mL}$ Schlenk tube equipped with a J. Young valve was frozen in liquid nitrogen, pumped, and backfilled with CO. The solution was stirred at $25{ }^{\circ} \mathrm{C}$ for $12 \mathrm{~h}$. After removal of the solvent under vacuum, the resulting dark brown solid was dissolved in hexane/pyridine (40:1) and crystallized at $-33^{\circ} \mathrm{C}$ to 3 as dark brown solid (62 $\mathrm{mg}, 0.0714 \mathrm{mmol}$, $58 \%$ ). Single crystals of $\mathbf{3}$ suitable for X-ray diffraction studies were obtained by recrystallization from a hexane/pyridine solution at $-33{ }^{\circ} \mathrm{C}$. The preparation of the ${ }^{13} \mathrm{C}$-enriched compound $\left[\left(\mathrm{C}_{5} \mathrm{Me}_{4} \mathrm{SiMe}_{3}\right)_{3} \mathrm{Ti}_{3}\left(\mu-\eta^{1}: \eta^{1}: \eta^{1}-{ }^{13} \mathrm{C}_{4} \mathrm{H}_{2} \mathrm{O}_{2}\right)\left(\mu_{2}-\mathrm{O}^{13} \mathrm{CH}_{3}\right)\left(\mu_{2}-\mathrm{O}\right)_{2}\right]\left(3^{13} \mathrm{C}_{5}\right)$ was carried out in the same manner by using ${ }^{13} \mathrm{CO}$.

3: ${ }^{1} \mathrm{H}$ NMR (400 MHz, THF- $d 8$, rt): 3.87 (s, 3H, OCH $H_{3}$ ), 3.83 (s, $1 \mathrm{H}, \mathrm{C}_{2} \mathrm{H}_{2} \mathrm{C}_{2} \mathrm{O}_{2}$ ), 3.49 (s, $1 \mathrm{H}$, $\left.\mathrm{C}_{2} \mathrm{H}_{2} \mathrm{C}_{2} \mathrm{O}_{2}\right), 2.37-1.48\left(\mathrm{~s}, 3 \mathrm{H} \times 12, \mathrm{C}_{5} \mathrm{Me}_{4} \mathrm{SiMe}_{3}\right), 0.21$ (s, 18H, $\left.\mathrm{C}_{5} \mathrm{Me}_{4} \mathrm{SiMe}_{3}\right), 0.17$ (s, 9H, $\mathrm{C}_{5} \mathrm{Me}_{4} \mathrm{SiMe}$ ). ${ }^{13} \mathrm{C}$ NMR (100 MHz, THF-d8, rt): $149.9\left(\mathrm{~s}, \mathrm{C}_{2} \mathrm{H}_{2} \mathrm{C}_{2} \mathrm{O}_{2}\right), 116.3\left(\mathrm{~s}, \mathrm{C}_{2} \mathrm{H}_{2} C_{2} \mathrm{O}_{2}\right), 134.8-$ 122.9 (s, C $C_{5} \mathrm{Me}_{4} \mathrm{SiMe}_{3}$ ), 119.8-112.4 (s, ipso- $C_{5} \mathrm{Me}_{4} \mathrm{SiMe}_{3}$ ), 95.5 (s, $C_{2} \mathrm{H}_{2} \mathrm{C}_{2} \mathrm{O}_{2}$ ), 85.1 (s, $\mathrm{C}_{2} \mathrm{H}_{2} \mathrm{C}_{2} \mathrm{O}_{2}$ ), 67.1 (s, $\mathrm{OCH}_{3}$ ), 15.9-11.5 (s, $\mathrm{C}_{5} \mathrm{Me}_{4} \mathrm{SiMe}_{3}$ ), 2.6-2.1 (s, $\mathrm{C}_{5} \mathrm{Me}_{4} \mathrm{SiMe}_{3}$ ). Anal. Calcd for $\mathrm{C}_{41} \mathrm{H}_{68} \mathrm{O}_{5} \mathrm{Si}_{3} \mathrm{Ti}_{3}: \mathrm{C}, 56.68 ; \mathrm{H}, 7.89$. Found: C, 56.42; H, 7.95.

3- $^{13} \mathrm{C}_{5}:{ }^{1} \mathrm{H}$ NMR $(100 \mathrm{MHz}, \mathrm{THF}-d 8, \mathrm{rt}): 3.87\left(\mathrm{~d}, J=143.2 \mathrm{~Hz}, \mathrm{O}^{13} \mathrm{CH}_{3}\right), 3.83(\mathrm{dd}, 1 \mathrm{H}, J=11.0$, $\left.188.0 \mathrm{~Hz},{ }^{13} \mathrm{C}_{2} \mathrm{H}_{2}{ }^{13} \mathrm{C}_{2} \mathrm{O}_{2}\right), 3.49\left(\mathrm{~s}, 1 \mathrm{H}, \mathrm{dd}, J=11.0,184.0 \mathrm{~Hz},{ }^{13} \mathrm{C}_{2} \mathrm{H}_{2}{ }^{13} \mathrm{C}_{2} \mathrm{O}_{2}\right), 2.37-1.48(\mathrm{~s}, 3 \mathrm{H} \times 12$, $\mathrm{C}_{5} \mathrm{Me}_{4} \mathrm{SiMe}_{3}$ ), 0.21 (s, 18H, $\left.\mathrm{C}_{5} \mathrm{Me}_{4} \mathrm{Si}_{2} e_{3}\right), 0.17$ (s, 9H, $\left.\mathrm{C}_{5} \mathrm{Me}_{4} \mathrm{Si}_{2} e_{3}\right) .{ }^{13} \mathrm{C} \mathrm{NMR}(100 \mathrm{MHz}, \mathrm{THF}-$ $d 8, \mathrm{rt}$ ) (partially characterized): 149.8 (ddd, $\left.J=47,43,10 \mathrm{~Hz},{ }^{13} \mathrm{C}_{2} \mathrm{H}_{2}{ }^{13} C_{2} \mathrm{O}_{2}\right), 116.2$ (ddd, $J=47$, $39,10 \mathrm{~Hz},{ }^{13} \mathrm{C}_{2} \mathrm{H}_{2}{ }^{13} C_{2} \mathrm{O}_{2}$ ), 95.5 (ddd, $J=43,33,10 \mathrm{~Hz},{ }^{13} \mathrm{C}_{2} \mathrm{H}_{2}{ }^{13} \mathrm{C}_{2} \mathrm{O}_{2}$ ), 85.1 (ddd, $J=39,33,10$ $\left.\mathrm{Hz},{ }^{13} \mathrm{C}_{2} \mathrm{H}_{2}{ }^{13} \mathrm{C}_{2} \mathrm{O}_{2}\right), 67.1\left(\mathrm{~s}, \mathrm{O}^{13} \mathrm{CH}_{3}\right)$. 


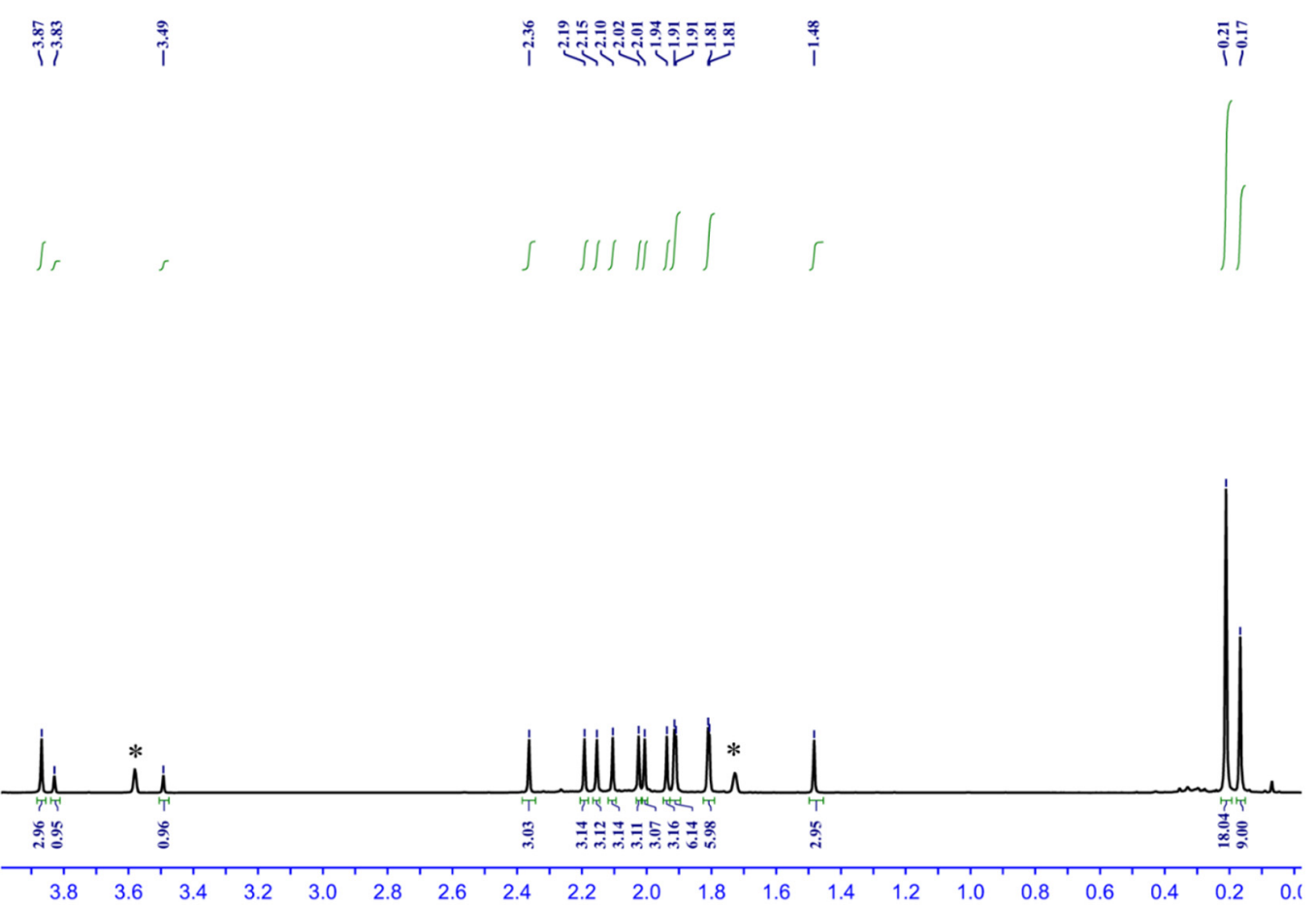

Figure S6. ${ }^{1} \mathrm{H}$ NMR spectrum of $\mathbf{3}\left(\mathrm{THF}-d_{8}\right.$, rt, $* \ldots$ residual internal $\left.\mathrm{C}_{4} \mathrm{D}_{7} \mathrm{HO}\right)$

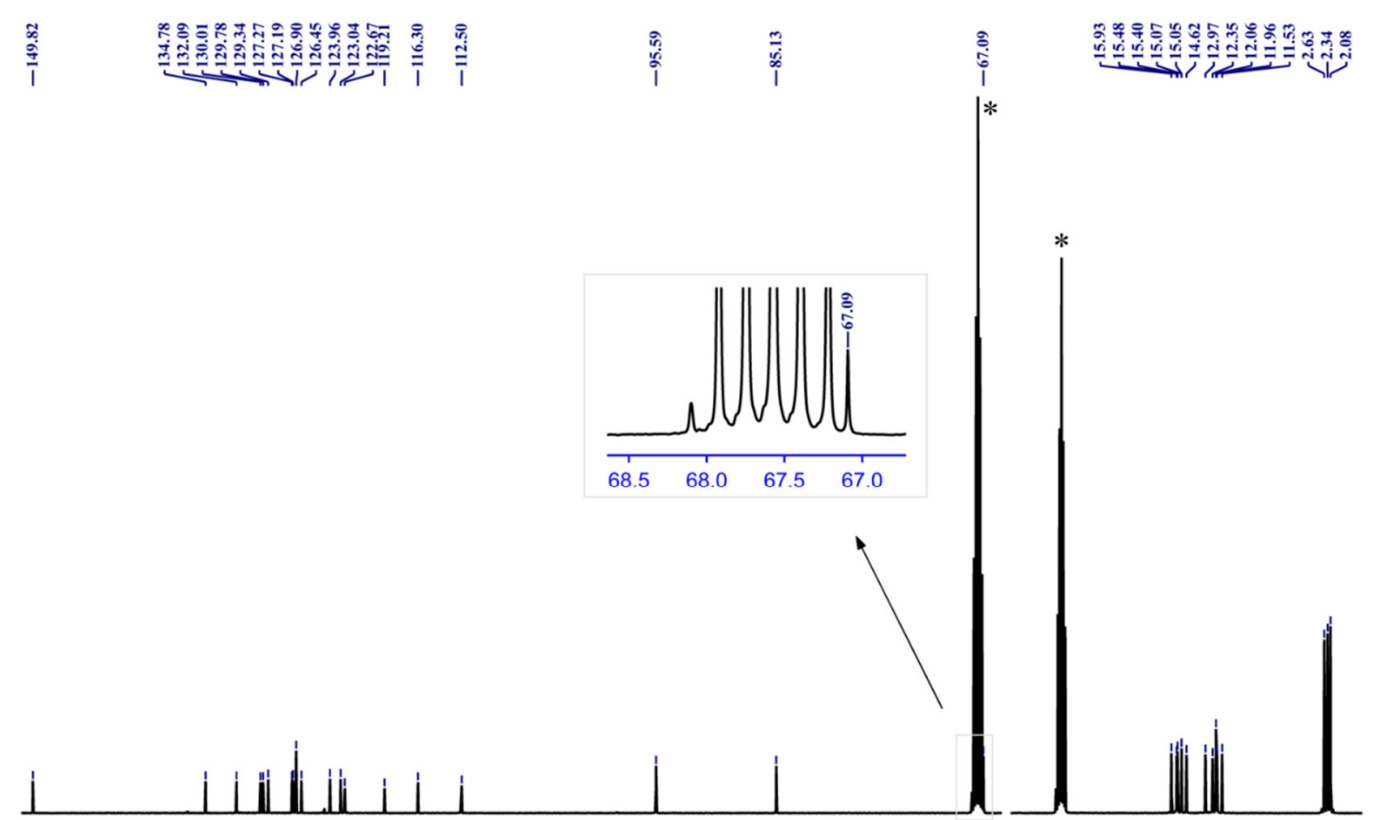

\begin{tabular}{llllllllllllllllllllllll}
\hline 50 & 145 & 140 & 135 & 130 & 125 & 120 & 115 & 110 & 105 & 100 & 95 & 90 & 85 & 80 & 75 & 70 & 1 & 25 & 20 & 15 & 10 & 5 & 0
\end{tabular}

Figure S7. ${ }^{13} \mathrm{C}$ NMR spectrum of $\mathbf{3}$ (THF- $\left.d_{8}, \mathrm{rt}, * \cdots \mathrm{THF}-d_{8}\right)$ 


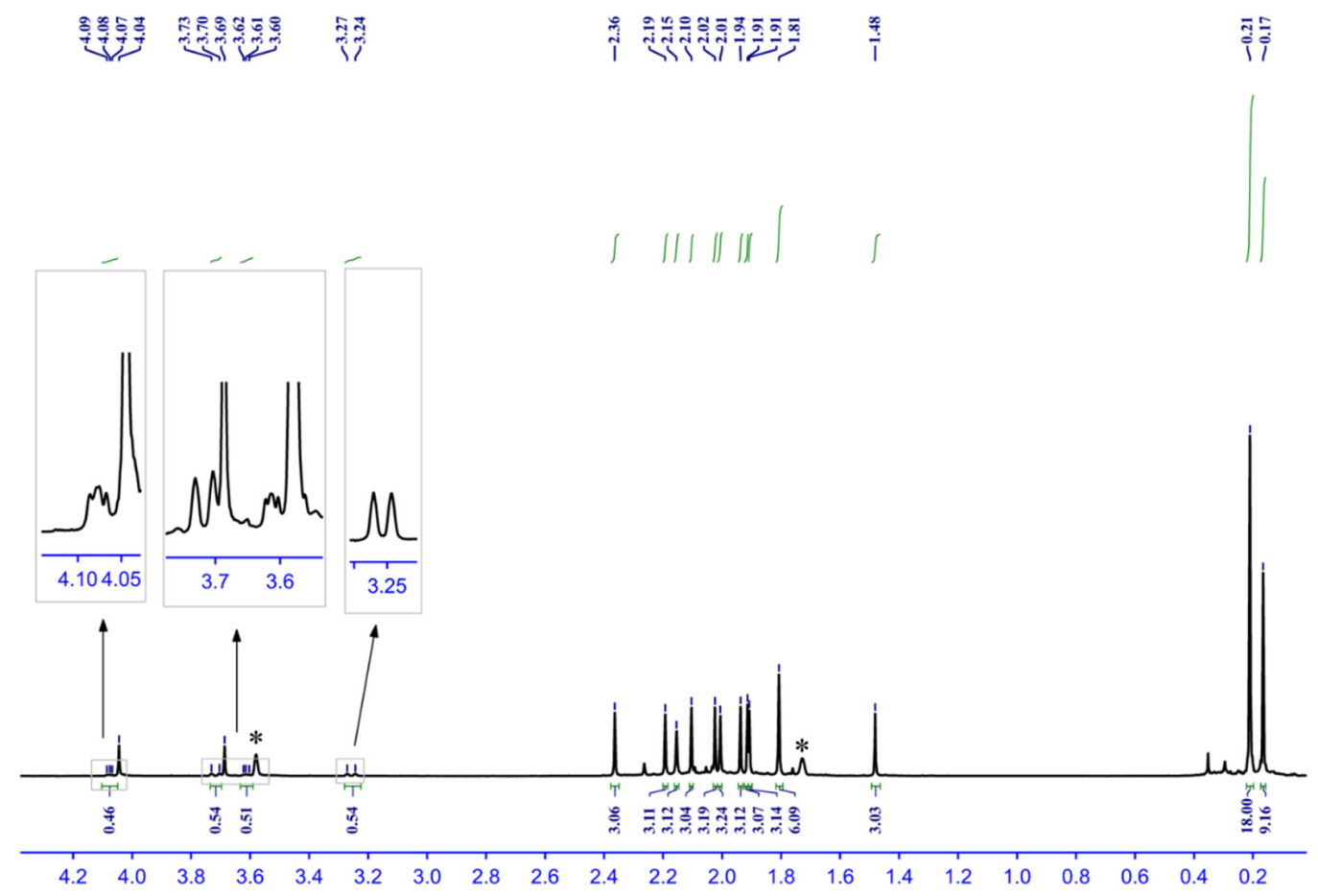

Figure S8. ${ }^{1} \mathrm{H}$ NMR spectrum of $\mathbf{3}^{-13} \mathbf{C}_{5}\left(\mathrm{THF}-d 8, \mathrm{rt}, * \cdots\right.$ residual internal $\left.\mathrm{C}_{4} \mathrm{D}_{7} \mathrm{HO}\right)$
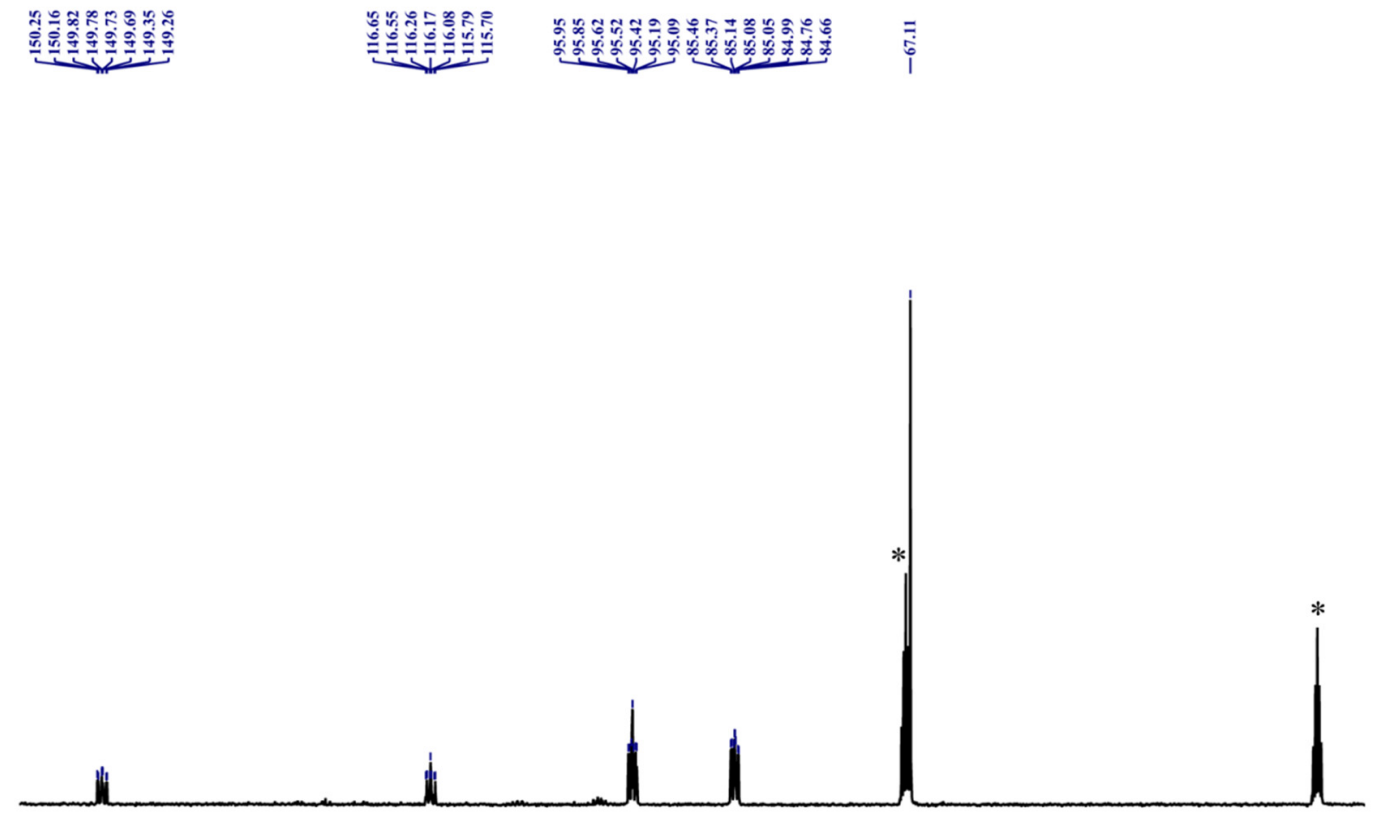

$\begin{array}{lllllllllllllllllllllllllll}155 & 150 & 145 & 140 & 135 & 130 & 125 & 120 & 115 & 110 & 105 & 100 & 95 & 90 & 85 & 80 & 75 & 70 & 65 & 60 & 55 & 50 & 45 & 40 & 35 & 30 & 25\end{array}$

Figure S9. ${ }^{13} \mathrm{C}$ NMR spectrum of $\mathbf{3}^{13} \mathbf{C}_{5}\left(\mathrm{THF}-d_{8}, \mathrm{rt}, * \cdots \mathrm{THF}-d_{8}\right)$ 
$\left[\left(\mathrm{C}_{5} \mathrm{Me}_{4} \mathrm{SiMe}_{3}\right)_{3} \mathrm{Ti}_{3}\left(\mu-\eta^{1}: \eta^{1}: \eta^{1}-\mathrm{C}_{4} \mathrm{H}_{4} \mathrm{O}_{2}\right)\left(\mu_{2}-\mathbf{O C H}_{3}\right)\left(\mu_{2}-\mathbf{O}\right)_{2}\right]$ (4). A hexane solution (3.0 mL) of 3 (80 mg, $0.0921 \mathrm{mmol}$ ) in $20 \mathrm{~mL}$ Schlenk tube equipped with a J. Young valve was frozen in liquid nitrogen, pumped, and backfilled with $\mathrm{H}_{2}(1 \mathrm{~atm})$. The solution was stirred at $60{ }^{\circ} \mathrm{C}$ for $12 \mathrm{~h}$. After removal of the solvent under vacuum, the resulting dark green solid was dissolved in DME and crystallized at $-33{ }^{\circ} \mathrm{C}$ to afford 4 as dark green solid (56 mg, $0.0643 \mathrm{mmol}, 70 \%$ ). Single crystals of 4 suitable for X-ray diffraction studies were obtained by recrystallization from a DME solution at $-33{ }^{\circ} \mathrm{C}$. The preparation of the ${ }^{13} \mathrm{C}$-enriched compound $\left[\left(\mathbf{C}_{5} \mathbf{M e}_{4} \mathbf{S i M e}_{3}\right)_{3} \mathbf{T i}_{3}\left(\mu-\eta^{1}: \eta^{1}: \eta^{1}-\right.\right.$ $\left.\left.{ }^{13} \mathrm{C}_{4} \mathrm{H}_{4} \mathrm{O}\right)\left(\boldsymbol{\mu}_{2}-\mathrm{O}^{13} \mathrm{CH}_{3}\right)\left(\boldsymbol{\mu}_{2}-\mathrm{O}\right)_{2}\right]\left({ }^{4-13} \mathrm{C}_{5}\right)$ was carried out in the same manner. 4 is paramagnetic complex. The partially deuterium-enriched compound $\left[\left(\mathbf{C}_{5} \mathbf{M e}_{4} \operatorname{SiMe}_{3}\right)_{3} \mathbf{T i}_{3}\left(\mu-\eta^{1}: \eta^{1}: \eta^{1}-\right.\right.$ $\left.\left.\mathrm{C}_{4} \mathrm{H}_{2} \mathrm{D}_{2} \mathrm{O}\right)\left(\boldsymbol{\mu}_{2}-\mathrm{OCH}\right)\left(\boldsymbol{\mu}_{2}-\mathbf{O}\right)_{2}\right]\left(\mathbf{( 4 - \boldsymbol { d } _ { 2 } )}\right.$ was synthesized through reaction of 3 with $\mathrm{D}_{2}(1 \mathrm{~atm})$. Complex 4 shows paramagnetic properties, which unable to be characterized by spectroscopic data. The accurate molecular weight has been confirmed by FD-MS.

4: FD-MS: $m / z=870.4\left([4]^{+}\right)$. Anal. Calcd for $\mathrm{C}_{41} \mathrm{H}_{70} \mathrm{O}_{5} \mathrm{Si}_{3} \mathrm{Tii}_{3}: \mathrm{C}, 56.55 ; \mathrm{H}, 8.10$. Found: $\mathrm{C}, 56.62$; H, 7.90.

4- $^{13} \mathbf{C}_{5}$ : FD-MS: $m / z=875.4\left(\left[4-{ }^{13} \mathbf{C}_{5}\right]^{+}\right)$.

4- $\boldsymbol{d}_{2}$ : FD-MS: $m / z=872.3\left(\left[\mathbf{4}-\boldsymbol{d}_{\mathbf{2}}\right]^{+}\right)$.

$\left[\left(\mathrm{C}_{5} \mathrm{Me}_{4} \mathrm{SiMe}_{3}\right)_{3} \mathrm{Ti}_{3}\left(\mu-\eta^{1}: \eta^{1}-\mathrm{C}_{4} \mathrm{H}_{4} \mathrm{O}\right)\left(\mu_{2}-\mathrm{OCH}_{3}\right)\left(\mu_{2}-\mathrm{O}\right)_{3}\right]$ (5). A benzene solution $(3.0 \mathrm{~mL})$ of 4 (75 mg, 0.0861 mmol) in a 30-mL Schlenk tube equipped with a J. Young valve was stirred at $100{ }^{\circ} \mathrm{C}$ for $3 \mathrm{~d}$. The color of the solution changed from dark green to dark red. After removal of the solvent under vacuum, the resulting dark red solid was dissolved in hexane and crystallized at $33{ }^{\circ} \mathrm{C}$ to afford $\mathbf{5}$ as dark red solid (68 mg, $\left.0.0781 \mathrm{mmol}, 91 \%\right)$. Single crystals of $\mathbf{5}$ suitable for X-ray diffraction studies were obtained by recrystallization from a hexane solution at $-33^{\circ} \mathrm{C}$. The 
preparation of the ${ }^{13} \mathrm{C}$-enriched compound $\left[\left(\mathbf{C}_{5} \mathbf{M e}_{4} \mathrm{SiMe}_{3}\right)_{3} \mathbf{T i}_{3}\left(\mu-\eta^{1}: \eta^{1-1}{ }^{13} \mathbf{C}_{4} \mathbf{H}_{4} \mathbf{O}\right)\left(\mu_{2}-\right.\right.$ $\left.\left.\mathbf{O}^{13} \mathbf{C H}_{3}\right)\left(\boldsymbol{\mu}_{2}-\mathbf{O}\right)_{3}\right]\left(\mathbf{5}^{13} \mathbf{C}_{5}\right)$ was carried out in the same manner.

5: ${ }^{1} \mathrm{H}$ NMR (400 MHz, $\left.\mathrm{C}_{6} \mathrm{D}_{6}, \mathrm{rt}\right): 4.06\left(\mathrm{~s}, 3 \mathrm{H}, \mathrm{OCH}_{3}\right), 2.88\left(\mathrm{~m}, 2 \mathrm{H}, \mathrm{C}_{4} \mathrm{H}_{4} \mathrm{O}\right), 2.60\left(\mathrm{~m}, 2 \mathrm{H}, \mathrm{C}_{4} \mathrm{H}_{4} \mathrm{O}\right)$, $2.29,2.26,2.20,2.18,2.12,2.07,2.05,2.03,1.93,1.90\left(\mathrm{~s}, 3 \mathrm{H} \times 8+6 \mathrm{H} \times 2, \mathrm{C}_{5} \mathrm{Me}_{4} \mathrm{SiMe}_{3}\right), 0.48(\mathrm{~s}$, 9H, $\left.\mathrm{C}_{5} \mathrm{Me}_{4} \mathrm{SiMe}_{3}\right), 0.49,0.46,0.45$ (s, 9H×3, $\left.\mathrm{C}_{5} \mathrm{Me}_{4} \mathrm{SiMe}_{3}\right) .{ }^{13} \mathrm{C} \mathrm{NMR}\left(100 \mathrm{MHz}, \mathrm{THF}-d_{8}, \mathrm{rt}\right):$ $186.3\left(\mathrm{~s}, C_{4} \mathrm{H}_{4} \mathrm{O}\right), 171.2\left(\mathrm{~s}, C_{4} \mathrm{H}_{4} \mathrm{O}\right), 131.6 .8-126.8$ (s, $\left.C_{5} \mathrm{Me}_{4} \mathrm{SiMe}_{3}\right), 122.4,119.4,119.2$ (s, ipso$\left.C_{5} \mathrm{Me}_{4} \mathrm{SiMe}_{3}\right), 62.8\left(\mathrm{~s}, \mathrm{OCH}_{3}\right), 35.2\left(\mathrm{~s}, \mathrm{C}_{4} \mathrm{H}_{4} \mathrm{O}\right), 28.1$ (s, $\left.\mathrm{C}_{4} \mathrm{H}_{4} \mathrm{O}\right), 15.2-11.3\left(\mathrm{~s}, \mathrm{C}_{5} \mathrm{Me}_{4} \mathrm{SiMe}_{3}\right), 2.2$, 2.2, 1.9 (s, $\mathrm{C}_{5} \mathrm{Me}_{4} \mathrm{Si}_{2} e_{3}$ ). Anal. Calcd for $\mathrm{C}_{41} \mathrm{H}_{70} \mathrm{O}_{5} \mathrm{Si}_{3} \mathrm{Ti}_{3}: \mathrm{C}, 56.55 ; \mathrm{H}, 8.10$. Found: $\mathrm{C}, 56.79 ; \mathrm{H}$, 8.05 .

5- ${ }^{13} \mathrm{C}_{5}:{ }^{1} \mathrm{H}$ NMR $\left(400 \mathrm{MHz}, \mathrm{C}_{6} \mathrm{D}_{6}, \mathrm{rt}\right): 4.05\left(\mathrm{~d}, J=140.5 \mathrm{~Hz}, 1 \mathrm{H}, \mathrm{O}^{13} \mathrm{CH}_{3}\right), 2.88\left(\mathrm{~m}, 2 \mathrm{H},{ }^{13} \mathrm{C}_{4} H_{4} \mathrm{O}\right)$, $2.60\left(\mathrm{~m}, 2 \mathrm{H},{ }^{13} \mathrm{C}_{4} \mathrm{H}_{4} \mathrm{O}\right), 2.29,2.26,2.20,2.18,2.12,2.07,2.05,2.04,1.93,1.90(\mathrm{~s}, 3 \mathrm{H} \times 8+6 \mathrm{H} \times 2$, $\left.\mathrm{C}_{5} \mathrm{Me}_{4} \mathrm{SiMe}_{3}\right), 0.48$ (s, 9H, $\left.\mathrm{C}_{5} \mathrm{Me}_{4} \mathrm{SiMe}_{3}\right), 0.49,0.46,0.45$ (s, 9H×3, $\left.\mathrm{C}_{5} \mathrm{Me}_{4} \mathrm{SiMe}_{3}\right) .{ }^{13} \mathrm{C} \mathrm{NMR}(100$ $\left.\mathrm{MHz}, \mathrm{C}_{6} \mathrm{D}_{6}, \mathrm{rt}\right)$ (partially characterized): $186.3\left(\mathrm{dd}, J=42,24 \mathrm{~Hz},{ }^{13} \mathrm{C}_{4} \mathrm{H}_{4} \mathrm{O}\right), 171.2(\mathrm{ddd}, J=42$, 34, $\left.8 \mathrm{~Hz},{ }^{13} \mathrm{C}_{4} \mathrm{H}_{4} \mathrm{O}\right), 62.7\left(\mathrm{~s}, \mathrm{O}^{13} \mathrm{CH}_{3}\right), 35.1\left(\mathrm{dd}, J=34,24 \mathrm{~Hz},{ }^{13} \mathrm{C}_{4} \mathrm{H}_{4} \mathrm{O}\right), 28.1(\mathrm{td}, J=24,8 \mathrm{~Hz}$, $\left.{ }^{13} \mathrm{C}_{4} \mathrm{H}_{4} \mathrm{O}\right)$. 


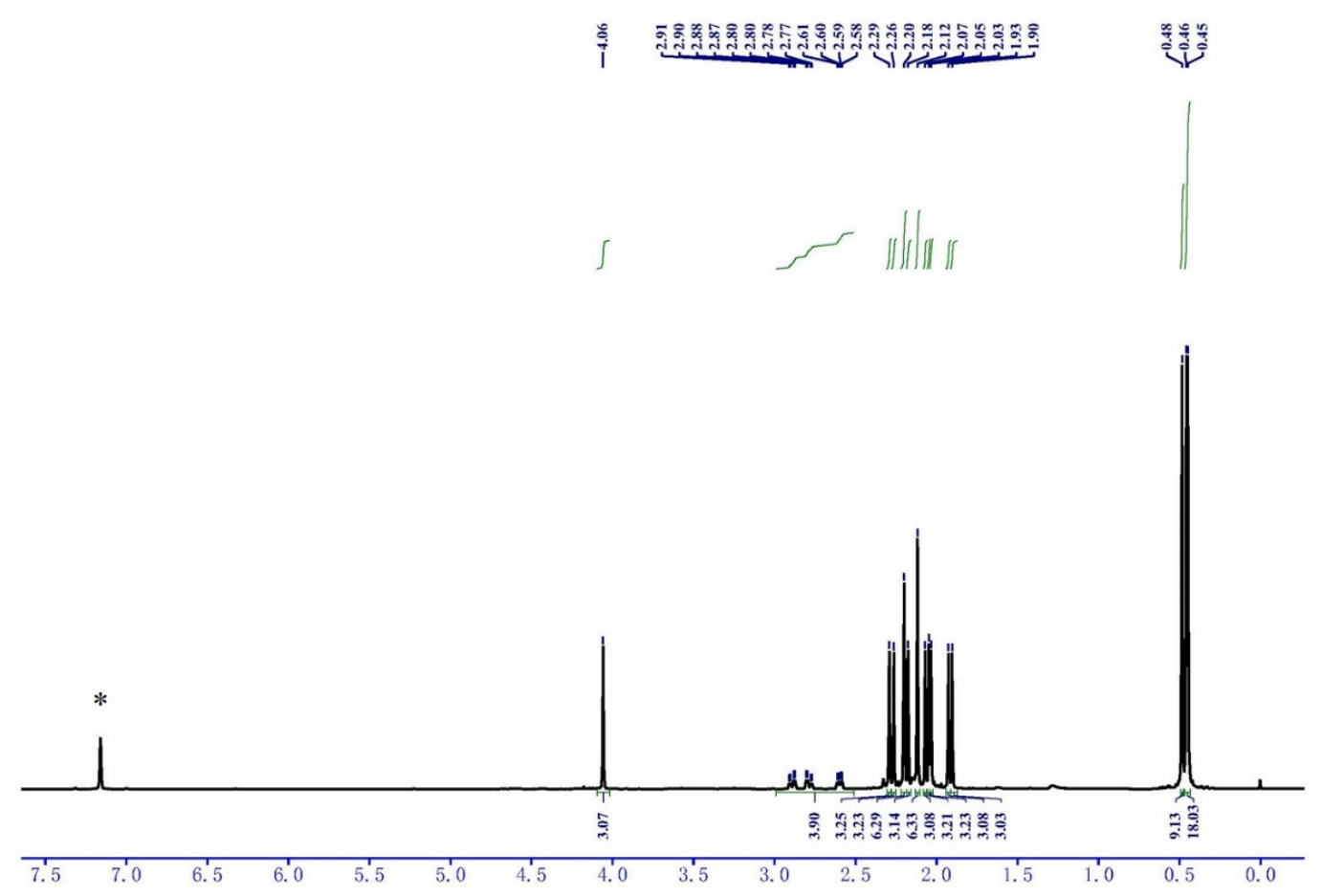

Figure S10. ${ }^{1} \mathrm{H}$ NMR spectrum of $5\left(\mathrm{C}_{6} \mathrm{D}_{6}, \mathrm{rt}, * \cdots\right.$ residual internal $\left.\mathrm{C}_{6} \mathrm{D}_{5} \mathrm{H}\right)$
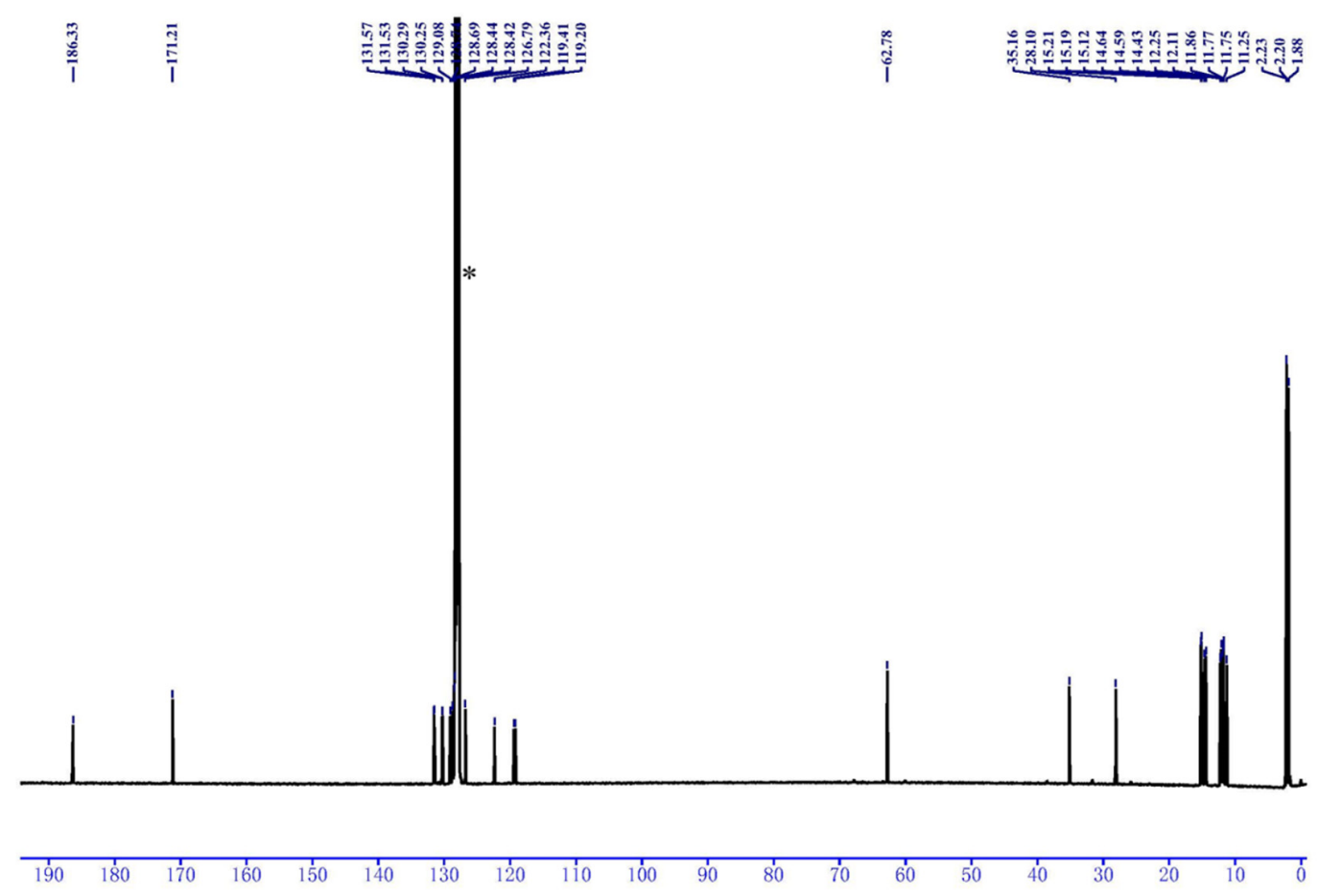

Figure S11. ${ }^{13} \mathrm{C}$ NMR spectrum of $5\left(\mathrm{C}_{6} \mathrm{D}_{6}, \mathrm{rt}, * \ldots \mathrm{C}_{6} \mathrm{D}_{6}\right)$ 


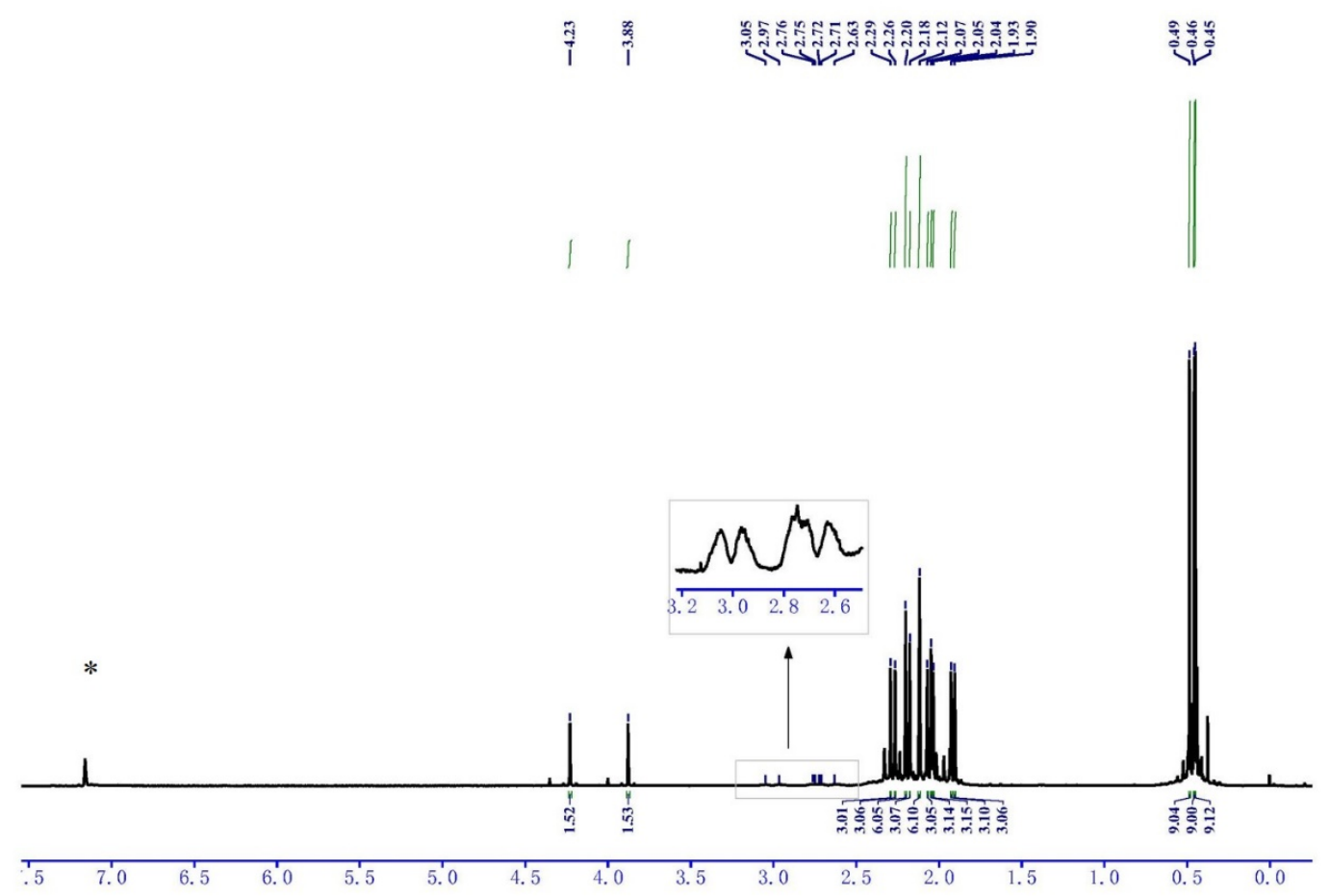

Figure S12. ${ }^{1} \mathrm{H}$ NMR spectrum of $\mathbf{5}_{-13}{ }^{13} \mathbf{C}_{5}\left(\mathrm{C}_{6} \mathrm{D}_{6}, \mathrm{rt},{ }^{*} \cdots\right.$ residual internal $\left.\mathrm{C}_{6} \mathrm{D}_{5} \mathrm{H}\right)$

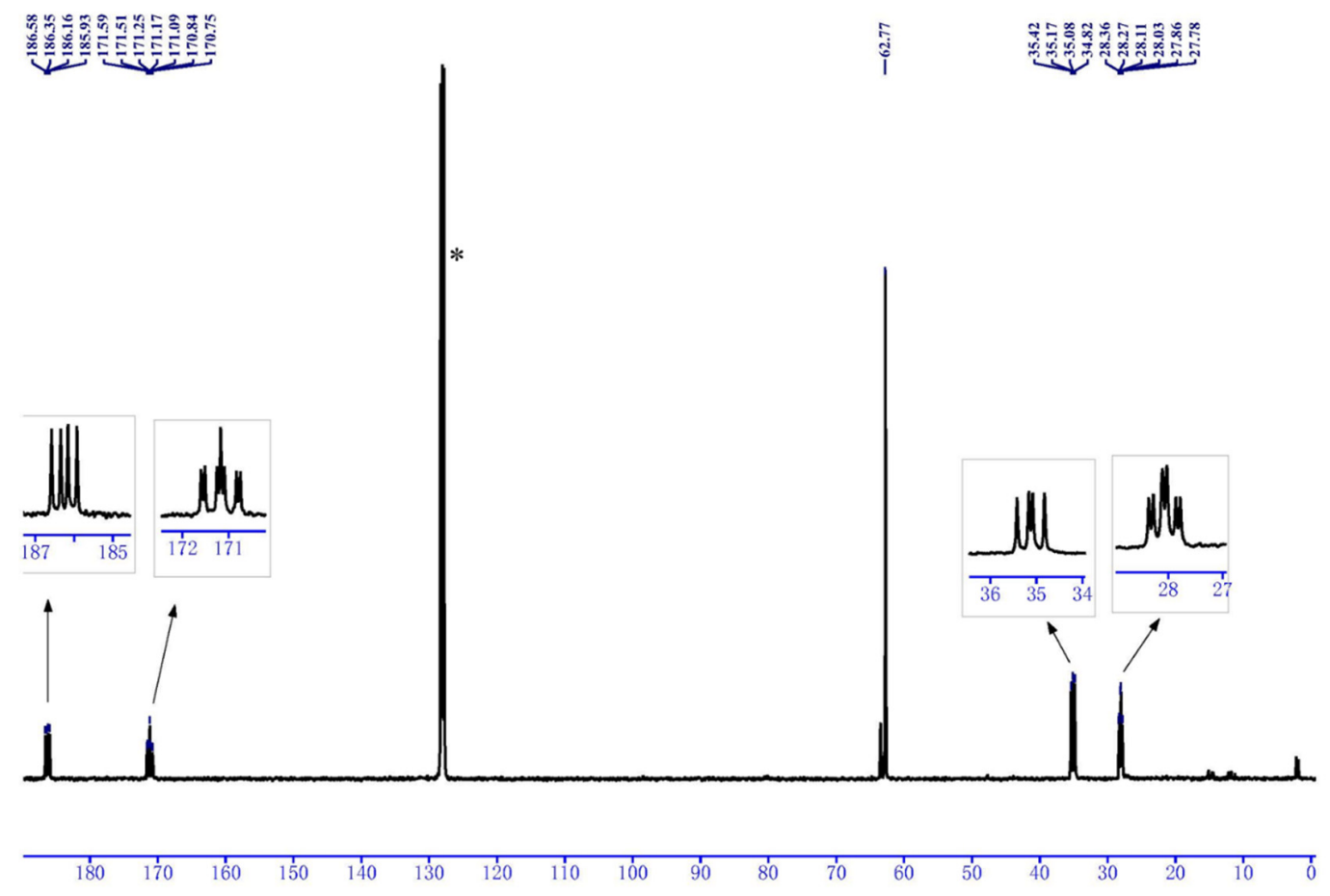

Figure S13. ${ }^{13} \mathrm{C}$ NMR spectrum of $\mathbf{5 -}^{13} \mathrm{C}_{5}\left(\mathrm{C}_{6} \mathrm{D}_{6}, \mathrm{rt},{ }^{*} \ldots \mathrm{C}_{6} \mathrm{D}_{6}\right)$ 


\section{FD-MS spectra}

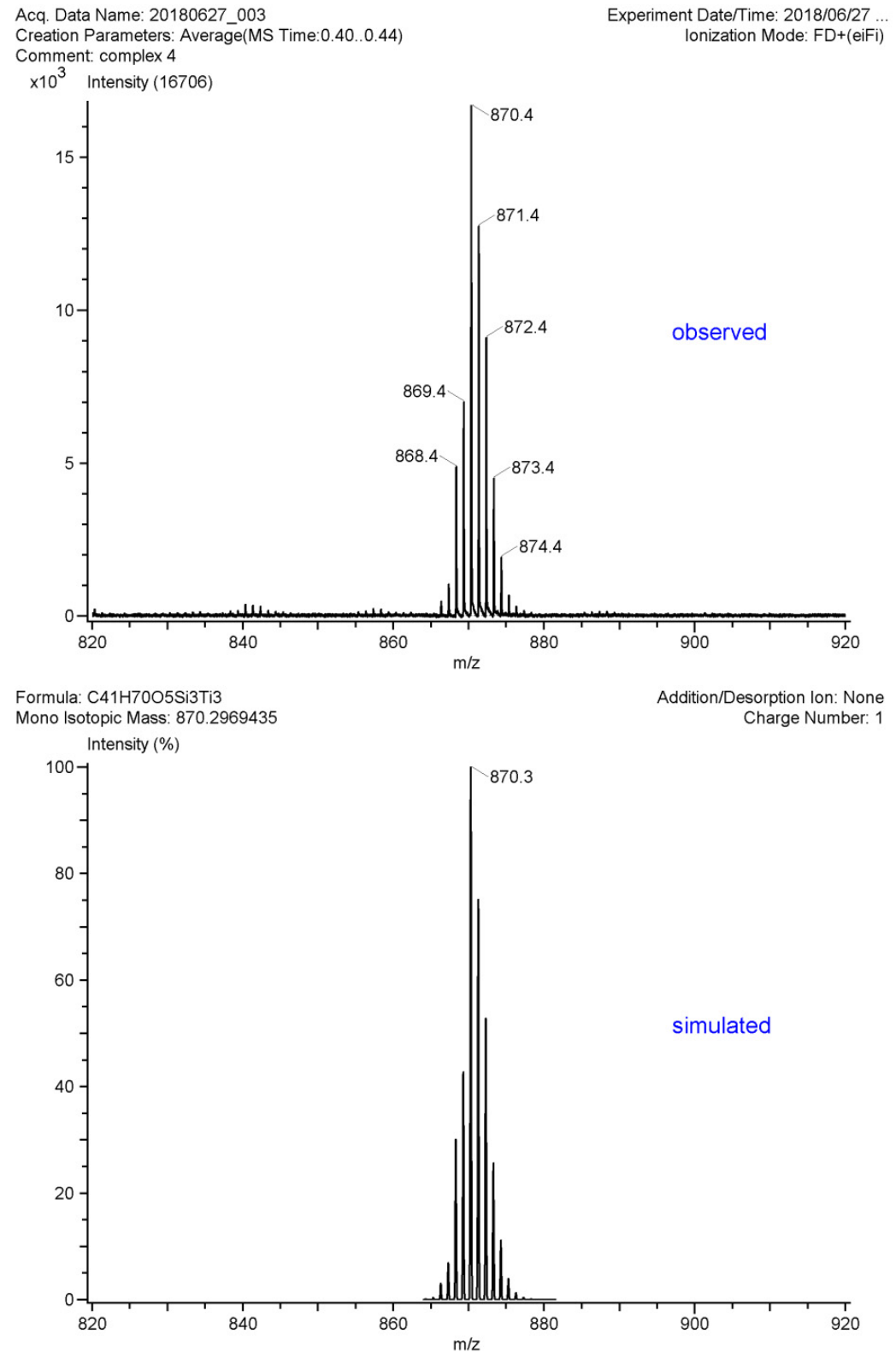

Figure S14. MS spectra of complex 4 (top: observed; bottom: simulated) 
Acq. Data Name: 20180627004

Creation Parameters: Average(MS Time:0.39..0.42)

Experiment Date/Time: 2018/06/27

Comment: complex 4-13C5

Ionization Mode: FD+(eiFi)

$\mathrm{x} 10^{3}$ Intensity (17483)

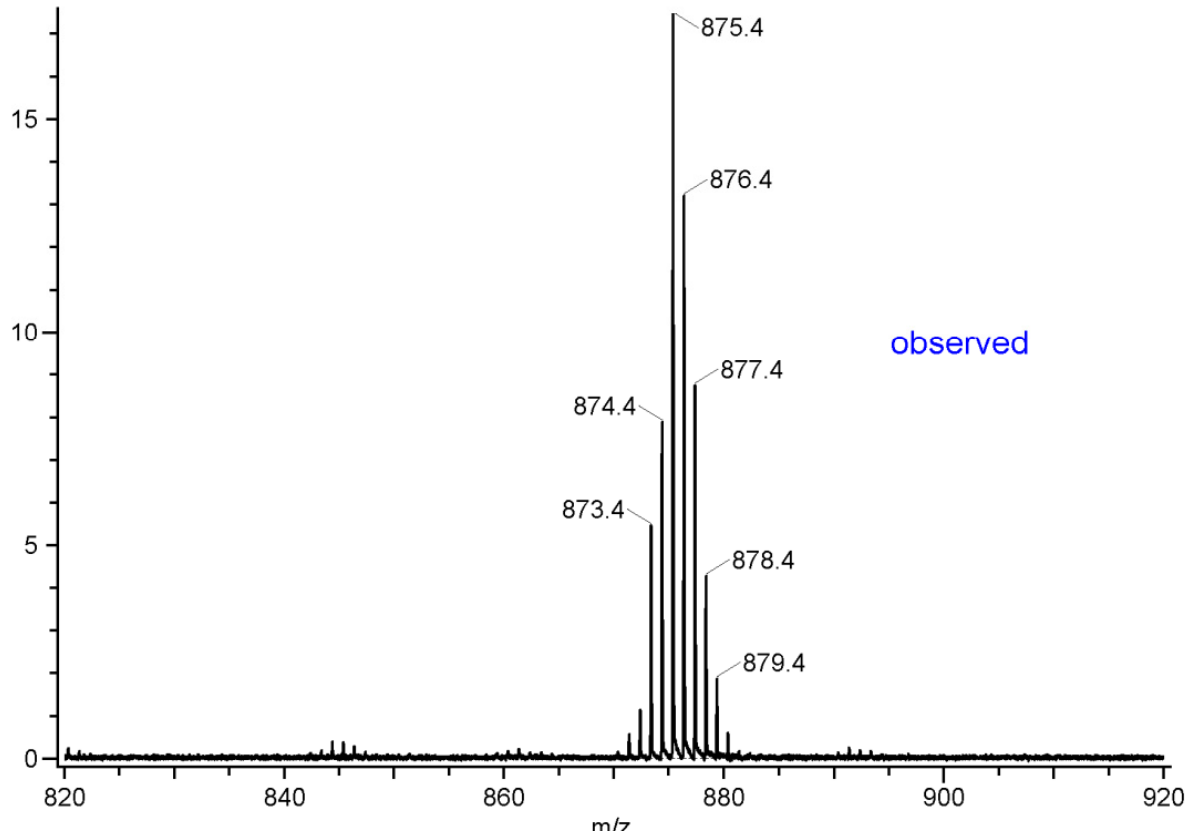

Figure S15. MS spectra of complex $4-{ }^{13} C_{5}$

Acq. Data Name: 20180727002

Creation Parameters: Average(MS Time:0.44..0.47)

Comment: complex 4-D2

$\times 10^{3}$ Intensity (214258)

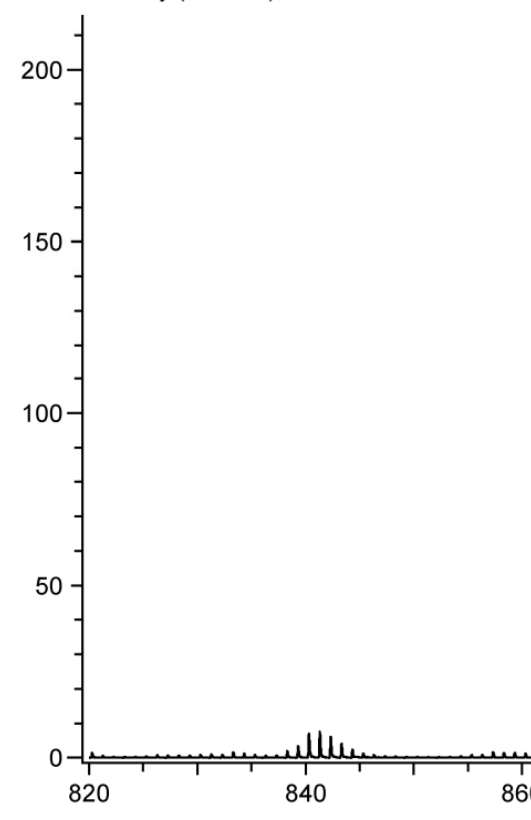

Experiment Date/Time: 2018/07/27 Ionization Mode: FD+(eiFi)

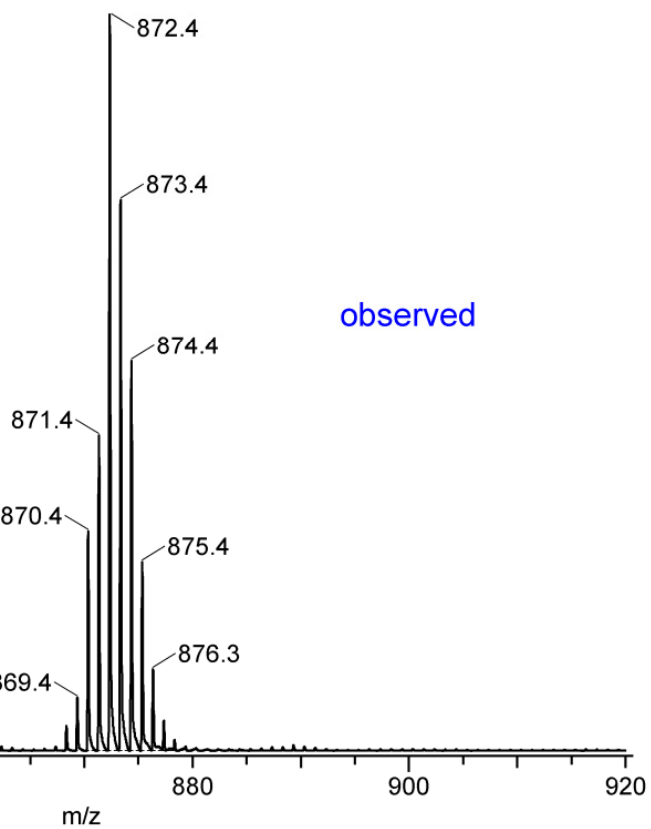

Figure S16. MS spectra of complex 4- $\boldsymbol{d}_{2}$ 


\section{Protonolysis Reactions}

Hydrochloric acid (3.0 M HCl aqueous solution, $61 \mu \mathrm{L}, 0.18 \mathrm{mmol})$ was added to a $\mathrm{C}_{6} \mathrm{D}_{6}$ solution ( $1.0 \mathrm{~mL})$ of $3(16 \mathrm{mg}, 0.018 \mathrm{mmol})$ via a syringe. After stirring for $12 \mathrm{~h}$, the reaction mixture was separated by a "trap-to-trap" technique. The volatile part was dried with $\mathrm{Na}_{2} \mathrm{SO}_{4}$. The organic products were confirmed by GC-MS and NMR analyses through comparison with authentic samples. The ${ }^{1} \mathrm{H}$ NMR integration against the 1,2,4,5-tetramethylbenzene internal standard showed $\gamma$-butyrolactone and methanol in 55\% and $45 \%$ yields, respectively. The residual orange-red solid was washed with cold hexane and dried under vacuum to give $\mathrm{Cp}^{\prime} \mathrm{TiCl}_{3}$ (16 mg, $0.046 \mathrm{mmol}, 85 \%$ yield). In a similar manner, the reaction of $\mathbf{3}^{-13} \mathbf{C}_{5}$ with hydrochloric acid afforded ${ }^{13} \mathrm{C}$-labelled $\gamma$-butyrolactone and methanol.

Similarly, the reaction of 5 with hydrochloric acid gave cyclobutanone (76\%), methanol (68\%), and $\mathrm{Cp}^{\prime} \mathrm{TiCl}_{3}(87 \%)$, respectively. 


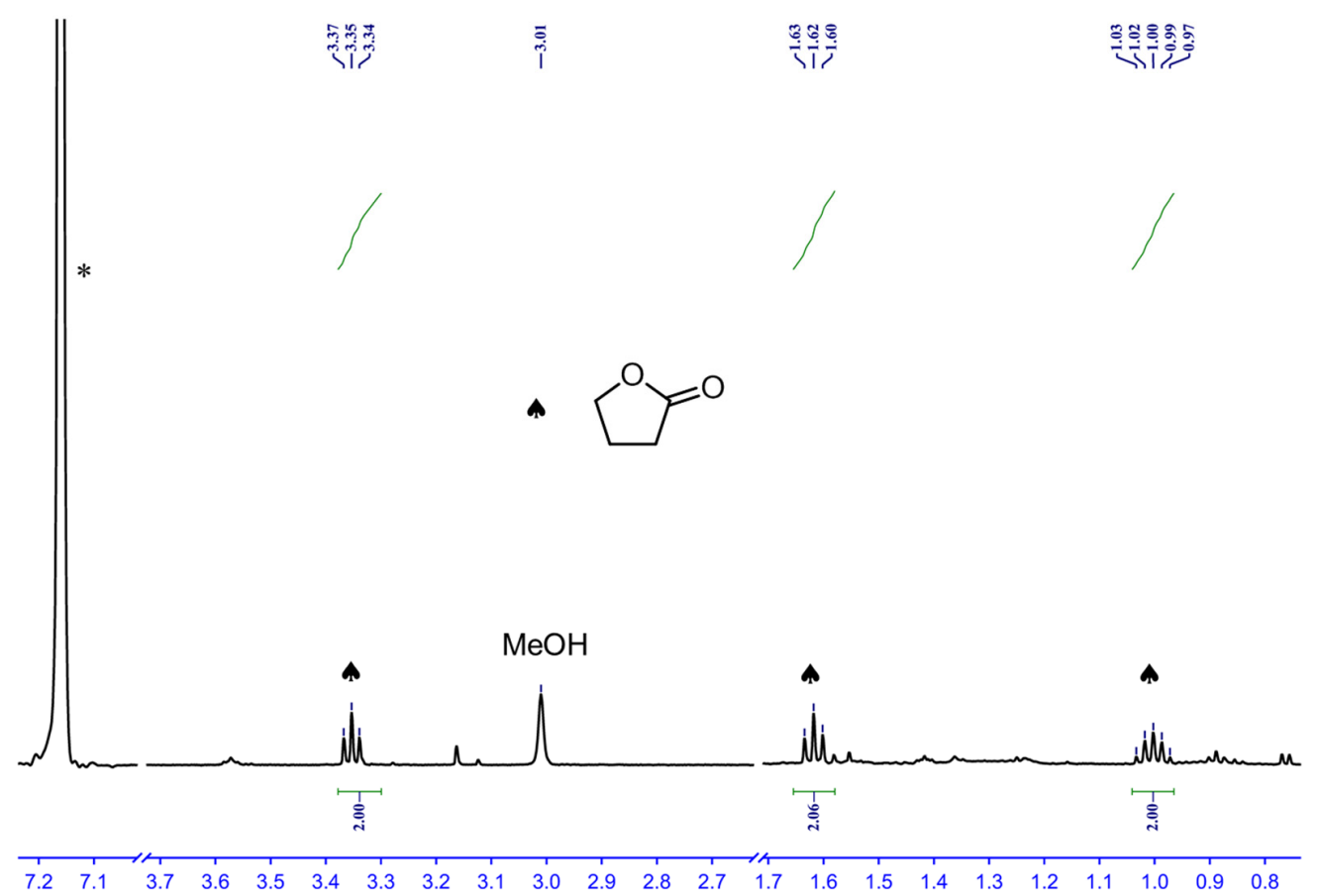

Figure S17. ${ }^{1} \mathrm{H}$ NMR spectrum of solvent part of 3 with hydrochloric acid $\left(\mathrm{C}_{6} \mathrm{D}_{6}, \mathrm{rt}, * \ldots\right.$ residual internal $\mathrm{C}_{6} \mathrm{D}_{5} \mathrm{H}$ )

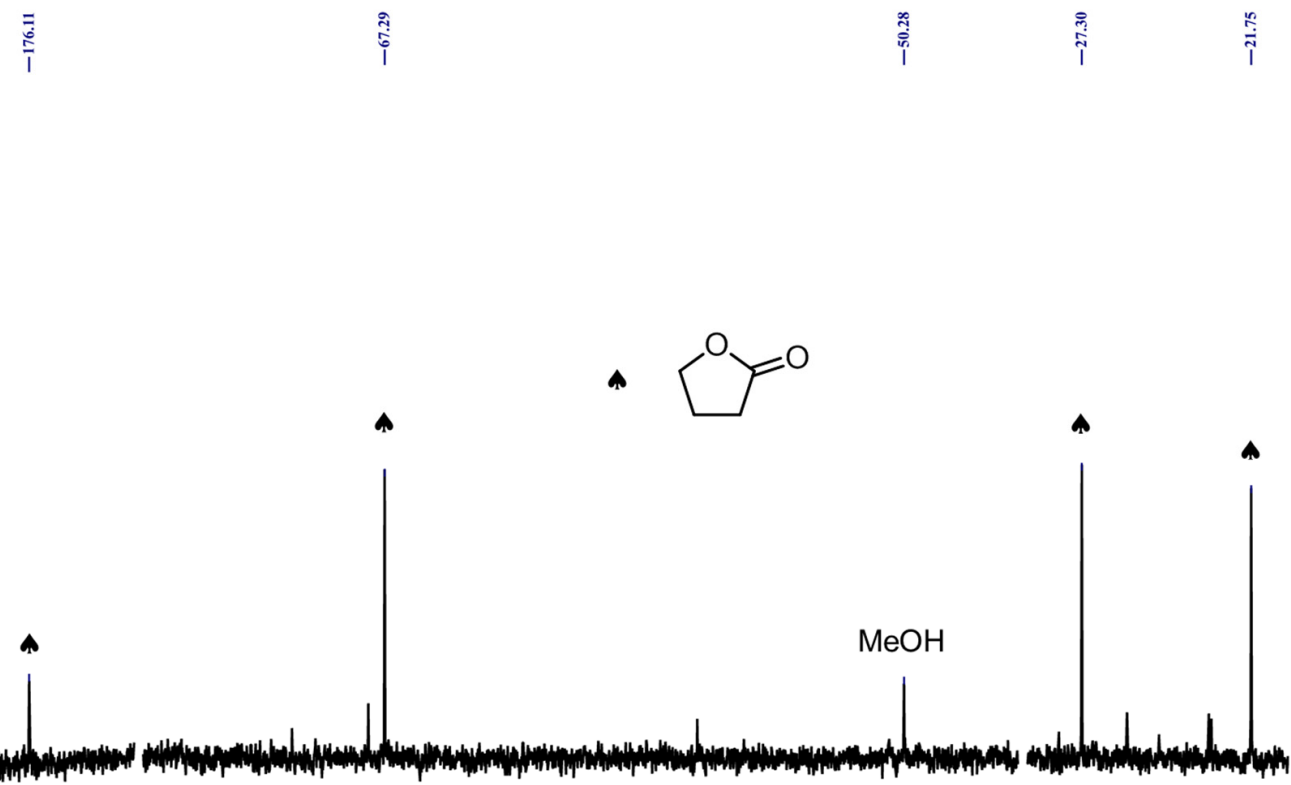

$77 \quad 175 \quad 1737574737271706968676665646362616059585756555453525150494847292827262524232221$

Figure S18. ${ }^{13} \mathrm{C}$ NMR spectrum of solvent part of 3 with hydrochloric acid $\left(\mathrm{C}_{6} \mathrm{D}_{6}, \mathrm{rt}\right)$ 

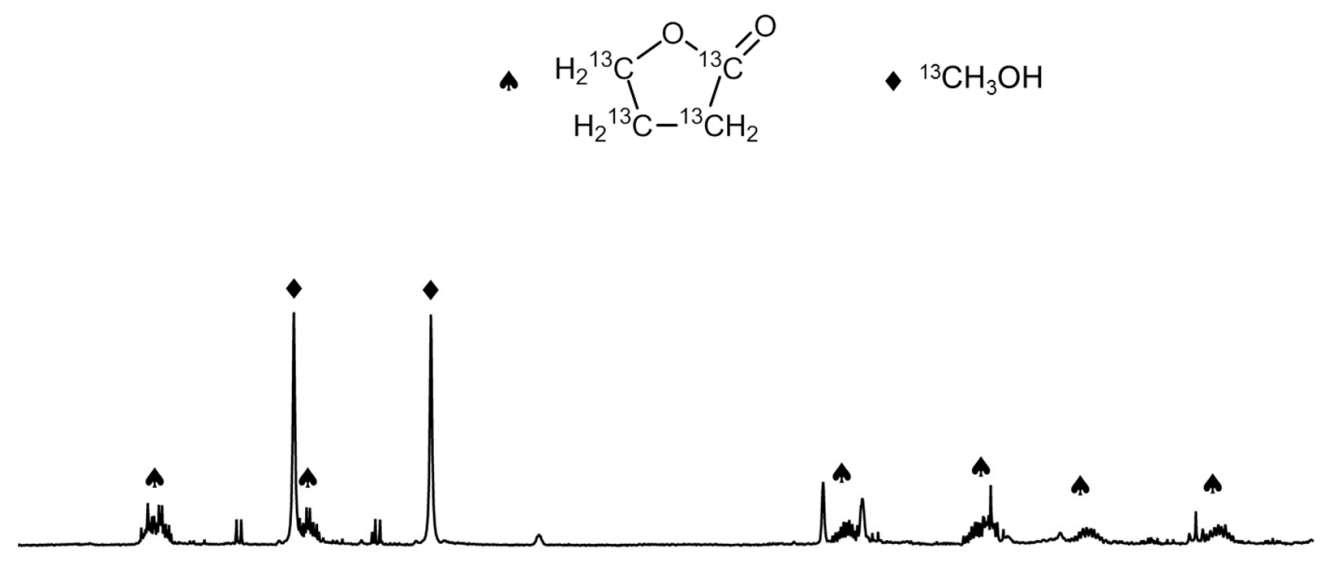

\begin{tabular}{llllllllllllllllllllllllllllllllllllllllll}
\hline 9 & 3.8 & 3.7 & 3.6 & 3.5 & 3.4 & 3.3 & 3.2 & 3.1 & 3.0 & 2.9 & 2.8 & 2.7 & 2.6 & 2.5 & 2.4 & 2.3 & 2.2 & 2.1 & 2.0 & 1.9 & 1.8 & 1.7 & 1.6 & 1.5 & 1.4 & 1.3 & 1.2 & 1.1 & 1.0 & 0.9 & 1 & 1.8 & 1 & 1 & 0.7 & 0.1
\end{tabular}

Figure S19. ${ }^{1} \mathrm{H}$ NMR spectrum of solvent part of $3-{ }^{13} \mathbf{C}_{5}$ with hydrochloric acid $\left(\mathrm{C}_{6} \mathrm{D}_{6}, \mathrm{rt}\right)$

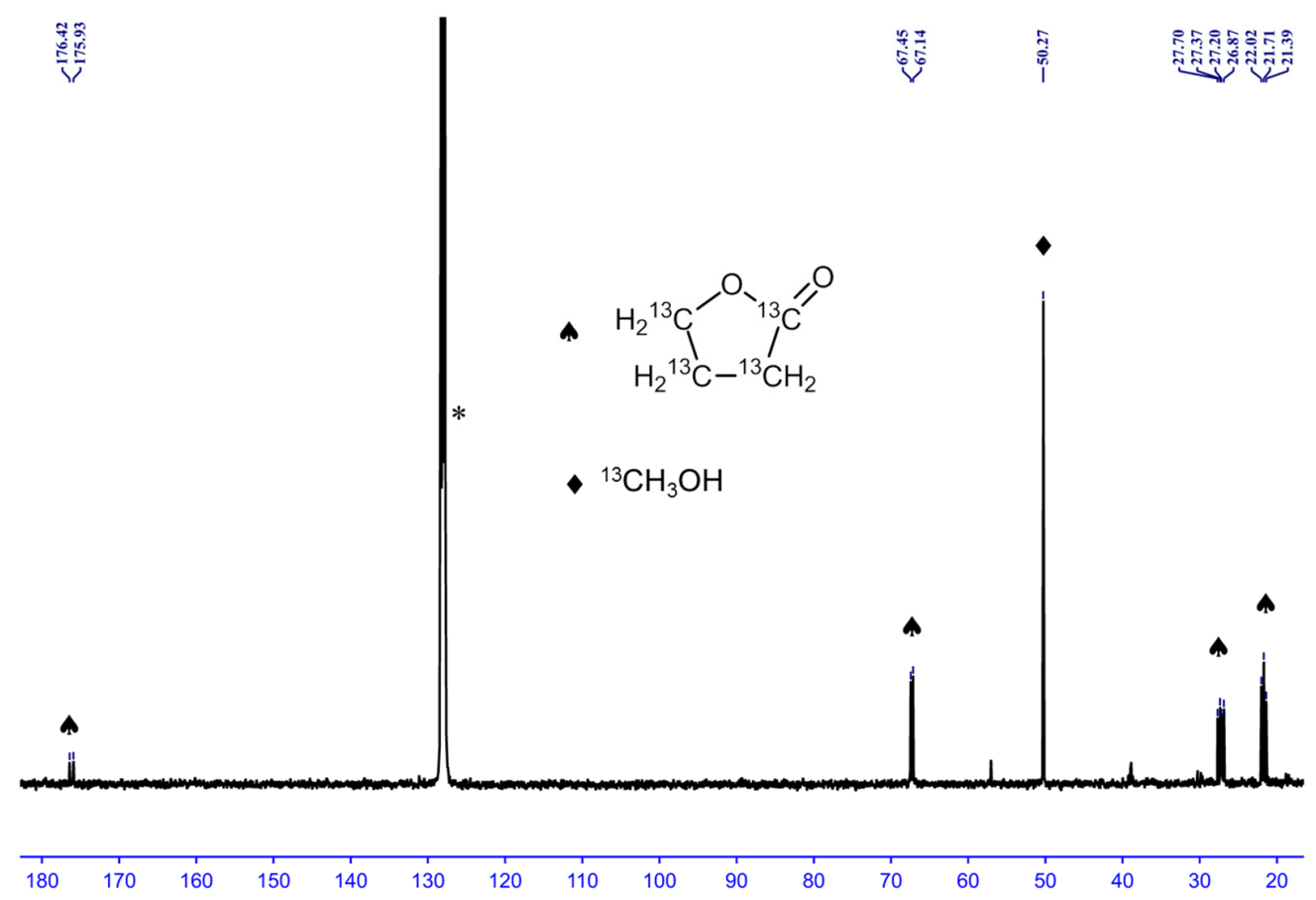

Figure S20. ${ }^{13} \mathrm{C}$ NMR spectrum of solvent part of $\mathbf{3 -}^{13} \mathbf{C}_{5}$ with hydrochloric acid $\left(\mathrm{C}_{6} \mathrm{D}_{6}, \mathrm{rt}\right.$, $* \ldots \mathrm{C}_{6} \mathrm{D}_{6}$ ) 


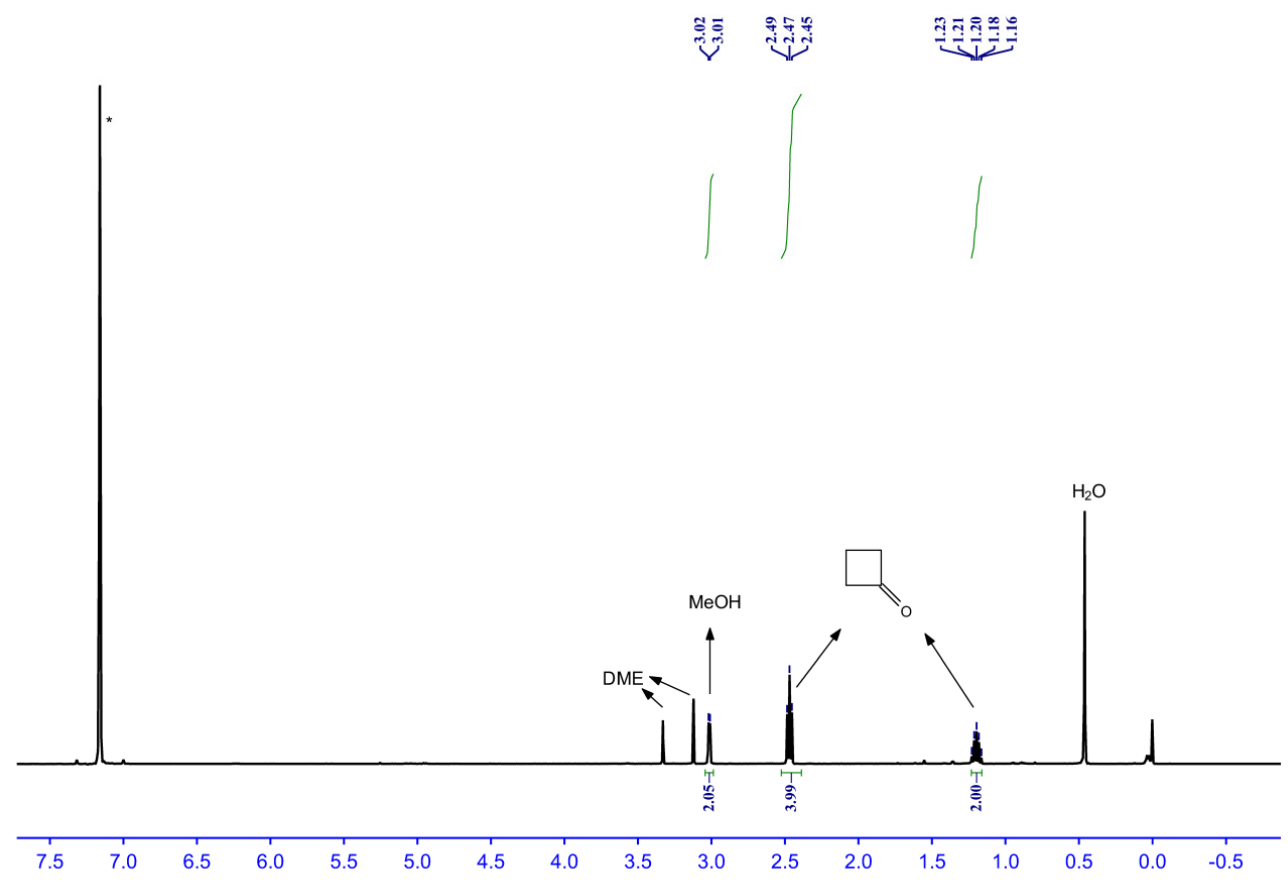

Figure S21. ${ }^{1} \mathrm{H}$ NMR spectrum of solvent part of 5 with hydrochloric acid $\left(\mathrm{C}_{6} \mathrm{D}_{6}, \mathrm{rt}, * \cdots\right.$ residual internal $\mathrm{C}_{6} \mathrm{D}_{5} \mathrm{H}, \mathrm{DME}$ from starting material 5)

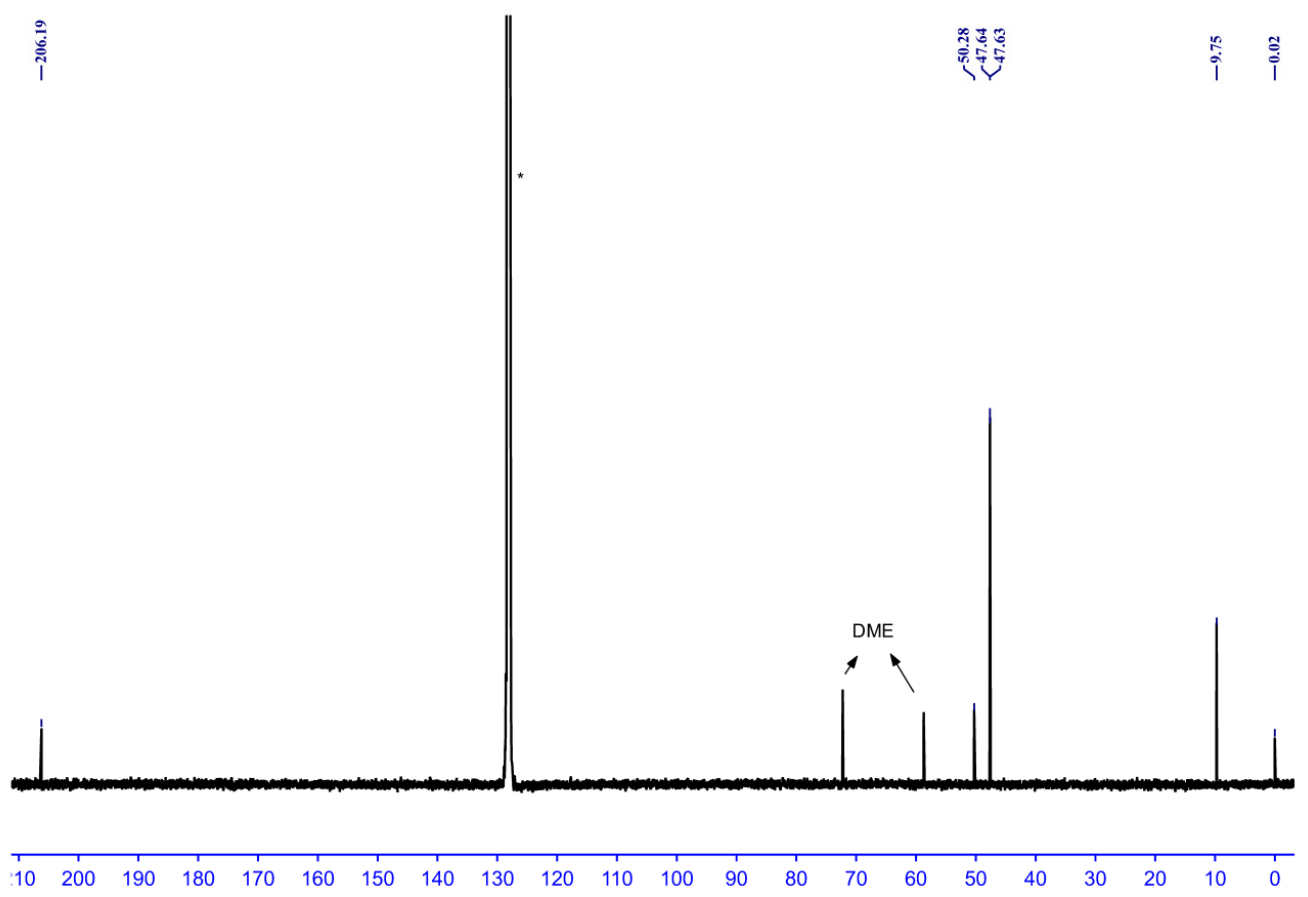

Figure S22. ${ }^{13} \mathrm{C}$ NMR spectrum of solvent part of 5 with hydrochloric acid $\left(\mathrm{C}_{6} \mathrm{D}_{6}, \mathrm{rt},{ }^{*}{ }^{*} \mathrm{C}_{6} \mathrm{D}_{6}\right)$ 


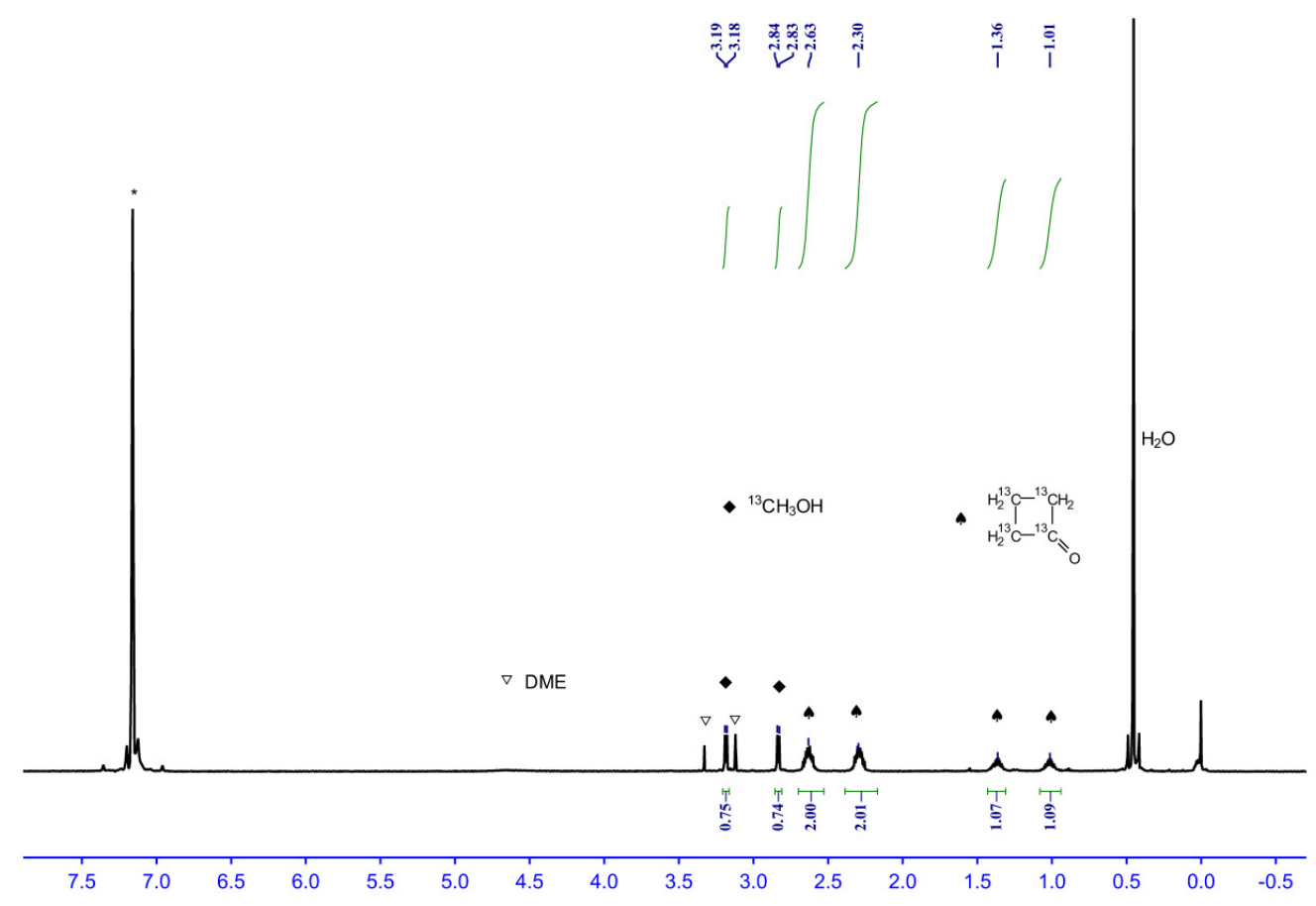

Figure S23. ${ }^{1} \mathrm{H}$ NMR spectrum of solvent part of $\mathbf{5 - 1}^{13} \mathbf{C}_{5}$ with acid $\left(\mathrm{C}_{6} \mathrm{D}_{6}, \mathrm{rt},{ }^{*} \cdots\right.$ residual internal $\mathrm{C}_{6} \mathrm{D}_{5} \mathrm{H}$ )

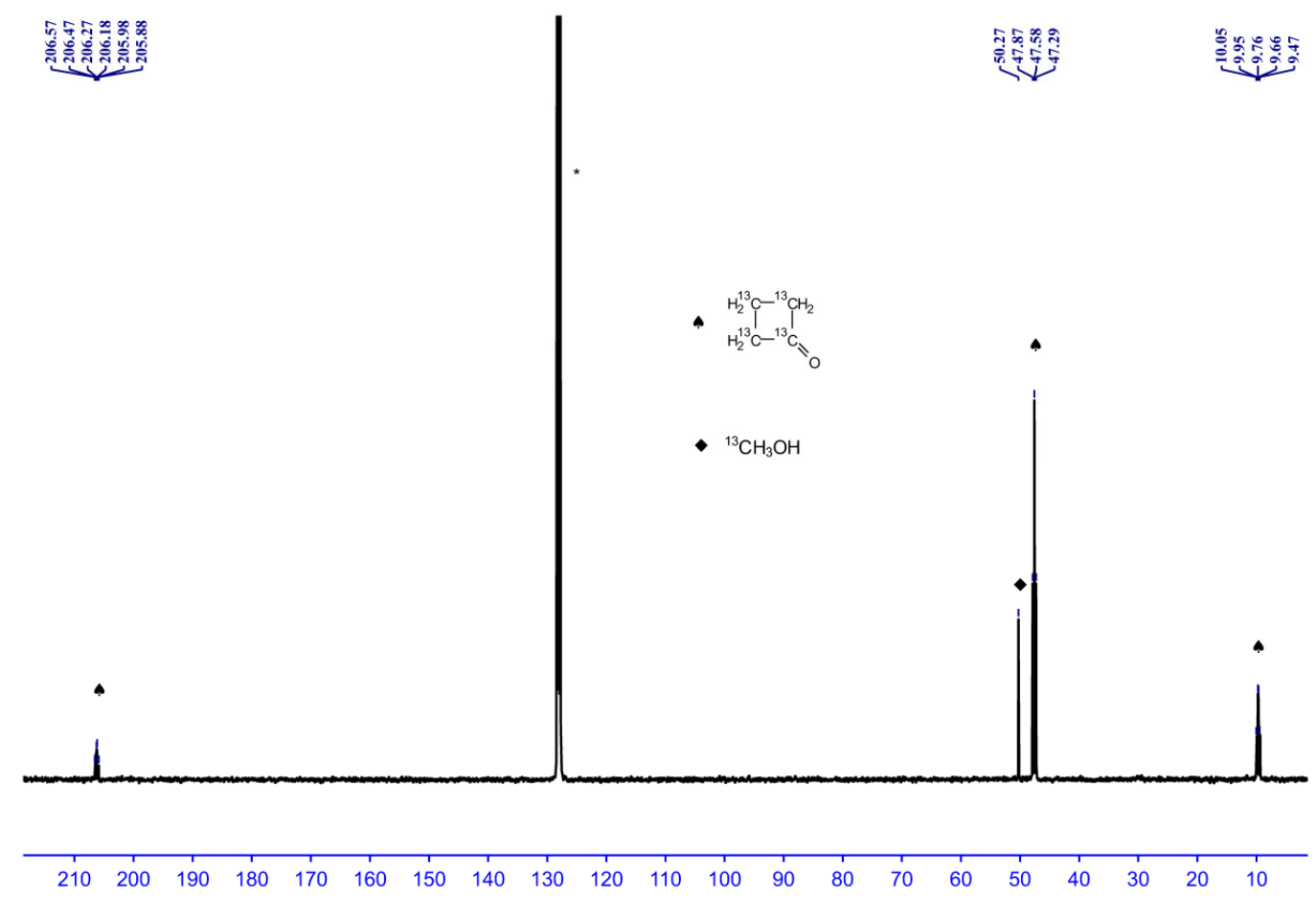

Figure S24. ${ }^{13} \mathrm{C}$ NMR spectrum of solvent part of $\mathbf{5}^{13} \mathrm{C}_{5}$ with acid $\left(\mathrm{C}_{6} \mathrm{D}_{6}, \mathrm{rt},{ }^{*} \cdots \mathrm{C}_{6} \mathrm{D}_{6}\right)$ 


\section{X-ray Crystallographic Studies}

Crystals for X-ray diffraction studies were obtained as described in the preparations. The crystals were manipulated in a glovebox under a microscope, and were sealed in thin-walled glass capillaries. X-ray diffraction data collections were performed on a Bruker D8 QUEST diffractometer equipped with a CMOS area detector, using a I $\mu \mathrm{S}$ (Incoatec Microfocus Source) microfocus sealed tube with Mo K $\alpha$ radiation $(\lambda=0.71073 \AA)$ at $173 \mathrm{~K}$. The Bravais lattice and the unit cell parameters were determined by the Bruker APEX3 software package. ${ }^{2}$ The raw frame data were processed, and absorption corrections were done using SAINT and SADABS embedded in Bruker APEX3 to yield the reflection data (hkl) file. ${ }^{2}$ All of the structures were solved using SHELXL-2018. ${ }^{3}$ Structural refinement was performed using on $\mathrm{F}^{2}$ anisotropically for all of the non-hydrogen atoms by the full matrix least-squares method. Structural refinement was performed using the SHELXL option in the WINGX system, ${ }^{4}$ or Olex2 system ${ }^{5}$ on $\mathrm{F}^{2}$ anisotropically for all of the non-hydrogen atoms by the full matrix least-squares method. Analytical scattering factors for neutral atoms were used throughout the analysis. The hydrogen atoms of the $\mathrm{Cp}^{\prime}$ ligands were placed at the calculated positions, which were refined using a riding model. Other hydrogen atoms could be located on difference Fourier maps and refined. The analytical scattering factors for neutral atoms were used throughout the analysis. The residual electron densities were of no chemical significance. 


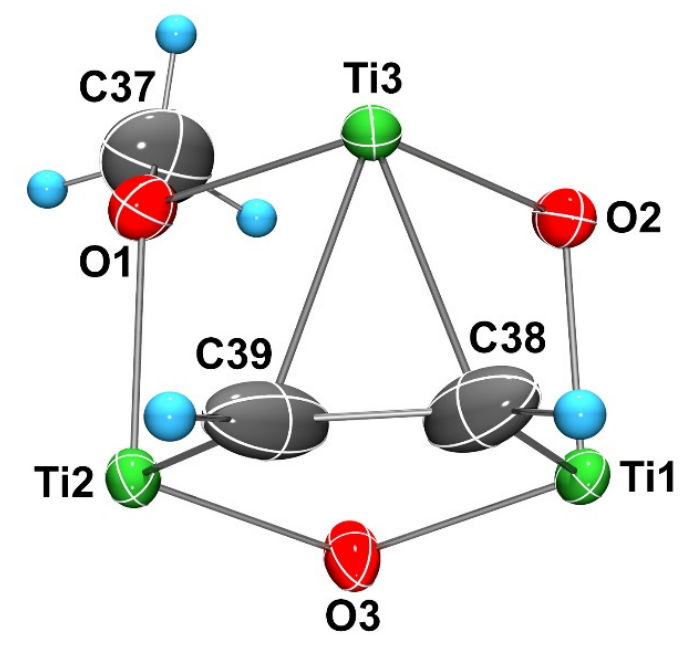

Figure S25. Solid state molecular structure of $\mathbf{2}$ at 30\% probability ellipsoids. $\mathrm{Cp}^{\prime}$ ligands are omitted for clarity.

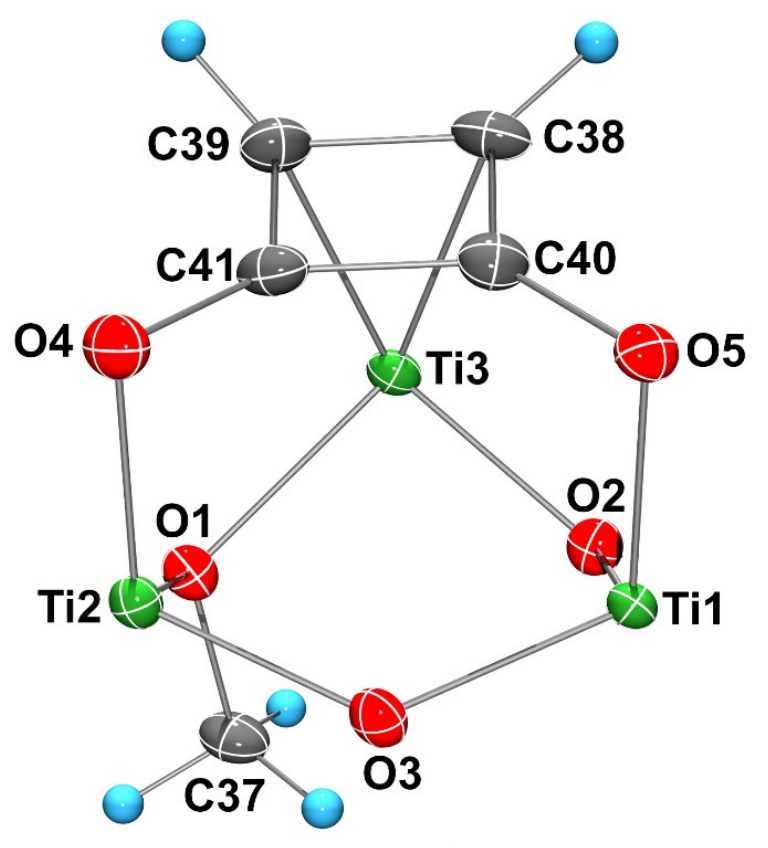

Figure S26. Solid state molecular structure of $\mathbf{3}$ at 30\% probability ellipsoids. Cp' ligands are omitted for clarity. 


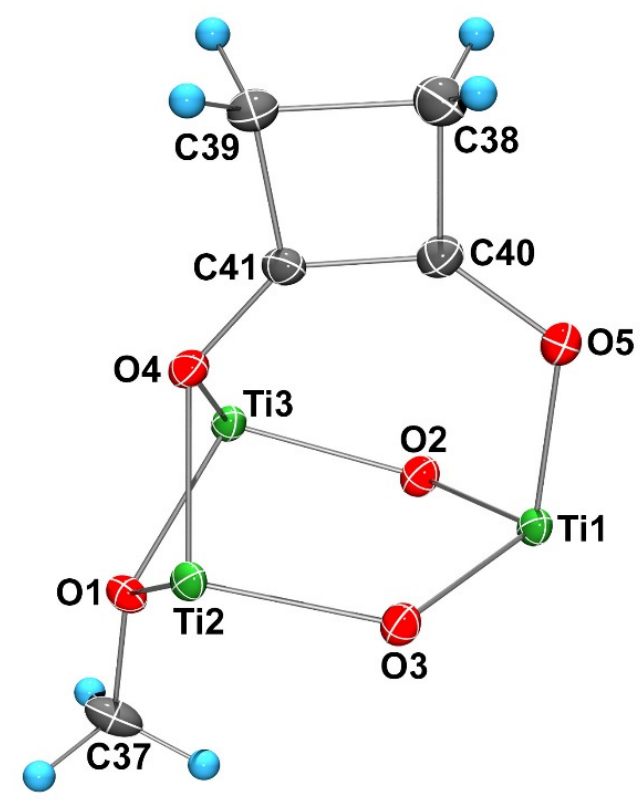

Figure S27. Solid state molecular structure of 4 at 30\% probability ellipsoids. Cp' ligands are omitted for clarity.

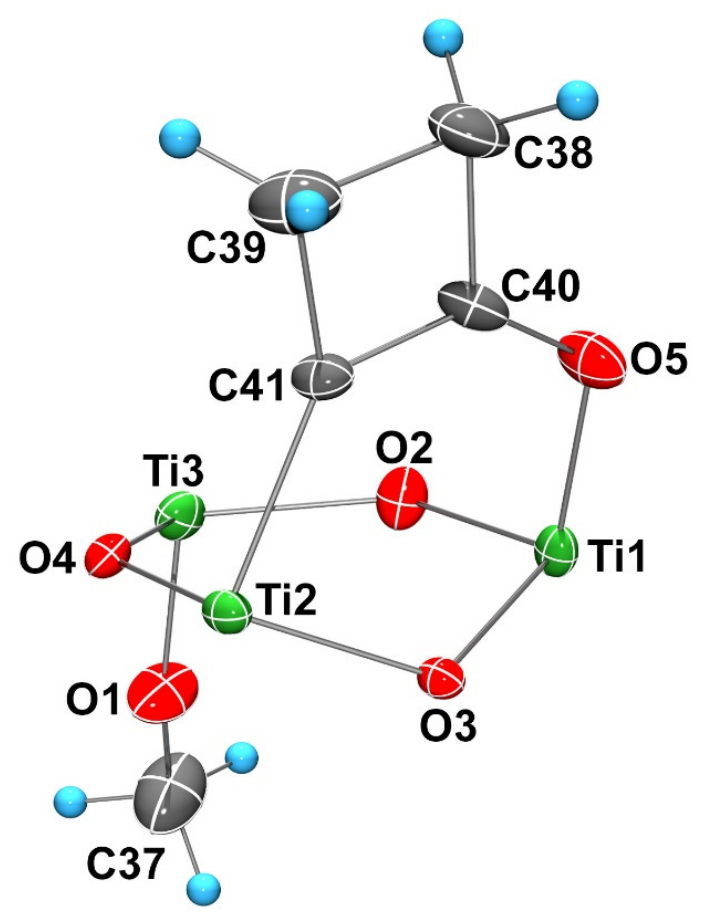

Figure S28. Solid state molecular structure of 5 at 30\% probability ellipsoids. Cp' ligands are omitted for clarity. 
Table S1. X-Ray crystallographic data and structure refinement for 2, 3, 4, and 5.

\begin{tabular}{|c|c|c|c|c|}
\hline & 2 & 3 & 4 & 5 \\
\hline formula & $\mathrm{C}_{39} \mathrm{H}_{68} \mathrm{O}_{3} \mathrm{Si}_{3} \mathrm{Ti}_{3}$ & $\mathrm{C}_{41} \mathrm{H}_{68} \mathrm{O}_{5} \mathrm{Si}_{3} \mathrm{Ti}_{3}$ & $\mathrm{C}_{41} \mathrm{H}_{70} \mathrm{O}_{5} \mathrm{Si}_{3} \mathrm{Ti}_{3}$ & $\mathrm{C}_{41} \mathrm{H}_{70} \mathrm{O}_{5} \mathrm{Si}_{3} \mathrm{Ti}_{3}$ \\
\hline formula weight & 812.81 & 868.83 & 870.85 & 870.85 \\
\hline crystal system & Triclinic & Triclinic & Triclinic & orthorhombic \\
\hline space group & $P-1$ & $P-1$ & $P 1$ & Pna $2_{1}$ \\
\hline $\mathrm{a}, \AA$ & $11.3762(3)$ & $8.7584(15)$ & $10.0717(17)$ & $20.8973(11)$ \\
\hline $\mathrm{b}, \AA$ & $12.0964(3)$ & $14.858(2)$ & $11.556(2)$ & $11.6751(6)$ \\
\hline $\mathrm{c}, \AA$ & $18.5162(5)$ & $20.540(4)$ & $12.004(2)$ & $19.2927(11)$ \\
\hline$\alpha, \operatorname{deg}$ & $98.867(1)$ & $70.651(5)$ & $61.262(6)$ & \\
\hline$\beta$, deg & $102.089(1)$ & $86.515(6)$ & $73.979(6)$ & \\
\hline$\gamma, \operatorname{deg}$ & $113.854(1)$ & $78.122(6)$ & $81.524(6)$ & \\
\hline$V, \AA^{3}$ & $2195.93(10)$ & 2467.9 (7) & $1177.2(4)$ & $4707.0(4)$ \\
\hline $\mathrm{Z}$ & 2 & 2 & 1 & 4 \\
\hline $\mathrm{D}_{\text {calcd }}, \mathrm{g} / \mathrm{cm}^{3}$ & 1.229 & 1.276 & 1.229 & 1.229 \\
\hline temp, $\mathrm{K}$ & $173(2)$ & $173(2)$ & $173(2)$ & $173(2)$ \\
\hline$\mu, \mathrm{mm}^{-1}(\mathrm{MoKa})$ & 0.65 & 0.59 & 0.61 & 0.61 \\
\hline reflections collected & 38362 & 42674 & 19765 & 34914 \\
\hline $\begin{array}{l}\text { independent reflections } \\
\left(R_{\text {int }}\right)\end{array}$ & $7759(0.033)$ & $8829(0.176)$ & $7455(0.061)$ & $9307(0.0370)$ \\
\hline$R 1(I>2 \sigma(I))$ & 0.0473 & 0.0609 & 0.0386 & 0.0587 \\
\hline$w R 2(I>2 \sigma(I))$ & 0.1209 & 0.1196 & 0.0926 & 0.1585 \\
\hline$w R 2$ (all data) & 0.1277 & 0.1482 & 0.0967 & 0.1611 \\
\hline parameters & 455 & 553 & 507 & 455 \\
\hline GOF & 1.039 & 0.975 & 1.006 & 1.036 \\
\hline absolute structure Flack & & & $0.030(16)$ & $0.012(9)$ \\
\hline
\end{tabular}




\section{References}

1. Shima, T.; Hu, S.; Luo, G.; Kang, X.; Luo, Y.; Hou, Z. Dinitrogen cleavage and hydrogenation by a trinuclear titanium polyhydride complex. Science 2013, 340, 1549-1552.

2. APEX3 v2016.1-0, Bruker AXS Inc., Madison, 2016.

3. G. M. Sheldrick, Acta Cryst. 2015, C71, 3-8.

4. L. J. J. Farrugia, WinGX suite for small-molecule single-crystal crystallography. J. Appl. Cryst. 1999, 32(4), 837-838.

5. O. V. Dolomanov, L. J. Bourhis, R. J. Gildea, J. A. K. Howard, H. Puschmann, J. Appl. Cryst. 2009, 42, 339-341. 